\title{
Cre-inducible human CD59 mediates rapid cell ablation after intermedilysin administration
}

\author{
Dechun Feng, ${ }^{1}$ Shen Dai, ${ }^{2,3}$ Fengming Liu, ${ }^{3}$ Yosuke Ohtake, ${ }^{4}$ Zhou Zhou, ${ }^{1}$ Hua Wang, ${ }^{1}$ Yonggang Zhang, ${ }^{3}$ Alison Kearns, ${ }^{3}$ \\ Xiao Peng, ${ }^{3}$ Faliang Zhu, ${ }^{2,3}$ Umar Hayat, ${ }^{4}$ Man Li, ${ }^{1}$ Yong He, ${ }^{1}$ Mingjiang $X u,{ }^{1}$ Chunling Zhao, ${ }^{3,5}$ Min Cheng, ${ }^{3,5}$ Lining Zhang, ${ }^{2}$ \\ Hong Wang, ${ }^{6}$ Xiaofeng Yang, ${ }^{6}$ Cynthia Ju, ${ }^{7}$ Elizabeth C. Bryda, ${ }^{8}$ Jennifer Gordon, ${ }^{3}$ Kamel Khalili, ${ }^{3}$ Wenhui Hu, ${ }^{3}$ Shuxin Li, ${ }^{4}$ \\ Xuebin Qin, ${ }^{3}$ and Bin Gao \\ 'Laboratory of Liver Diseases, National Institute on Alcohol Abuse and Alcoholism (NIAAA), NIH, Bethesda, Maryland, USA. 'Department of Immunology, Shandong University School of Medicine, \\ Jinan, China. ${ }^{3}$ Department of Neuroscience and ${ }^{4}$ Shriners Hospitals Pediatric Research Center, Department of Anatomy and Cell Biology, Temple University School of Medicine, \\ Philadelphia, Pennsylvania, USA. ${ }^{5}$ Key Laboratory for Immunology in Universities of Shandong Province, Weifang Medical University, Weifang, China. ${ }^{6}$ Centers of Metabolic Disease Research and \\ Cardiovascular Research, Temple University School of Medicine, Philadelphia, Pennsylvania, USA. ${ }^{7}$ Skaggs School of Pharmacy and Pharmaceutical Sciences and Integrated Immunology, \\ University of Colorado Anschutz Medical Campus, Aurora, Colorado, USA. ${ }^{8}$ Rat Resource and Research Center and Department of Veterinary Pathobiology, University of Missouri, Columbia, Missouri, USA
}

Cell ablation is a powerful tool for studying cell lineage and/or function; however, current cell-ablation models have limitations. Intermedilysin (ILY), a cytolytic pore-forming toxin that is secreted by Streptococcus intermedius, lyses human cells exclusively by binding to the human complement regulator CD59 (hCD59), but does not react with CD59 from nonprimates. Here, we took advantage of this feature of ILY and developed a model of conditional and targeted cell ablation by generating floxed STOP-CD59 knockin mice (ihCD59), in which expression of human CD59 only occurs after Cre-mediated recombination. The administration of ILY to ihCD59+ mice crossed with various Cre-driver lines resulted in the rapid and specific ablation of immune, epithelial, or neural cells without off-target effects. ILY had a large pharmacological window, which allowed us to perform dose-dependent studies. Finally, the ILY/ihCD59-mediated cell-ablation method was tested in several disease models to study immune cell functionalities, hepatocyte and/or biliary epithelial damage and regeneration, and neural cell damage. Together, the results of this study demonstrate the utility of the ihCD59 mouse model for studying the effects of cell ablation in specific organ systems in a variety of developmental and disease states.

\section{Introduction}

Conditional and targeted cell ablation is a powerful and widely used approach for studying specific cellular functions as well as tissue repair and differentiation in vivo $(1,2)$. The genetic cell-ablation methods that are currently used by researchers include the expression of herpes simplex virus 1 thymidine kinase (HSVtk) and the diphtheria toxin (DT) receptor (DTR) coupled with transgenic strategies (1-3). However, these approaches have some limitations, restraining their broader application in biomedical research. For example, in the model of HSVtk transgenic mice, only dividing cells are eliminated, whereas nondividing cells are not ablated (4). Although the DTR cell-ablation model has been used in the study of cellular functionalities in vivo for more than 15 years $(1,2)$, it also has limitations. Several groups have recently reported that DT administration of only 2- to 3-fold higher doses than the effective doses required for targeted cell ablation results in significant offtarget effects, including local lung and renal toxicity and significant weight loss, causing mortality and morbidity independent of DTR (5-7). Because of these observed toxicities, DT injection to wildtype mice has even been proposed as a model for studying experi-

Authorship note: D. Feng, S. Dai, and F. Liu are co-first authors and contributed equally to this work.

Conflict of interest: The authors have declared that no conflict of interest exists. Submitted: October 2, 2015; Accepted: March 3, 2016.

Reference information: / Clin Invest. 2016;126(6):2321-2333. doi:10.1172/JCI84921. mental podocyte injury (7). The narrow pharmacological dose window of the DT-mediated cell-ablation model often makes it difficult to distinguish target effects from off-target effects upon DT delivery in DTR transgenic mice. These facts underscore an unmet need to develop a new model that specifically ablates cells in vivo with higher efficiency and fewer off-target effects.

Intermedilysin (ILY) is a cholesterol-dependent cytolysin (CDC) that is secreted by Streptococcus intermedius. ILY can specifically lyse human cells by binding to human CD59 (hCD59) and subsequently forming toxin pores; however, ILY does not lyse cells from 9 other animal species that were tested because there is no cross-species reactivity of ILY with the CD59 from these species $(8,9)$, even when the ILY concentration was 5,000-fold greater than the dose that yields the concentration of toxin required to lyse $50 \%$ of the human erythrocytes $\left(\mathrm{HD}_{50}\right)$. These 9 species include cat, chicken, cow, dog, horse, rabbit, sheep, rat, and mouse (8). CD59 is a glycosylphosphatidylinositol-linked (GPI-linked) membrane protein that inhibits the formation of the membrane attack complex of complement by binding to complement proteins C8 and C9 and preventing C9 incorporation and polymerization (10). We previously developed 2 lines of $h$ CD59 transgenic mice that express hCD59 specifically in erythrocytes or endothelial cells (11). No obvious adverse phenotypes were observed in these transgenic mice. The injection of ILY causes massive erythrocyte and endothelial damage in erythrocyte- and endothelial-specific hCD59 transgenic mice, respectively, indicating that ILY is able 
to efficiently and specifically lyse hCD59-expressing cells in mice in vivo $(11,12)$. This result suggests that ILY-mediated cell killing might provide an alternative approach to specifically ablating cells in vivo; however, the potential broad application of the ILY-mediated cell-ablation model has not been explored. In the current paper, we generated a line of Cre-inducible floxed STOP-hCD59 transgenic mice, where specific hCD59 expression occurs following Cre-mediated recombination (ihCD59). By crossing ihCD59 with transgenic mice that express Cre in a cell-specific manner or by delivering an adenovirus expressing Cre, we obtained several lines of mice in which ihCD59 was specifically expressed in a spatially regulated manner on the surface of immune cells, epithelial cells, or neural cells. ILY injection resulted in conditionally specific cell ablation in various types of cells without any detectable off-target effects on nontargeted cell populations, including the adjacent tissue cells. Moreover, we tested this ablation technique in various disease models and found that this model is valuable for the study of cellular functionalities, tissue injury and regeneration, and neural injury.

\section{Results}

Generation of ihCD59 transgenic mice and ILY-mediated immune cell ablation. LoxP-Stop-loxP-hCD59 (LSL-hCD59) knockin (ihCD59) mice were generated using TARGATT Technology (Applied Stem Cell), as described in the Supplemental Experimental Procedures (supplemental material available online with this article; doi:10.1172/JCI84921DS1), with a transgenic construct in which the $h C D 59$ gene was placed downstream of the CAG promoter and loxP-STOP cassette-loxP element (pCAG-LSL-hCD59) (Figure 1A). Briefly, the construct was verified by in vitro transfection experiments showing that the cells transfected with the construct expressed hCD59 on the surface upon adding Cre-recombinase, but did not express hCD59 without Cre expression (Supplemental Figure 1). Then the construct was introduced into the H11 locus by pronuclear injection to generate ihCD59 knockin mice at mouse genomic locus H11 (Figure 1A), and the Cre-inducible hCD59 expression in ihCD59 mice was generated by crossing ihCD59 mice with both a germline expressing Cre and cell-specific Cre transgenic lines (Figure 1B).

The ihCD59 mice were crossed with Meox2-Cre transgenic mice (a germline expressing Cre line) to investigate whether expression from the $\mathrm{H} 11$ locus was uniform in all cell types. As illustrated in Supplemental Figure 2, A-C, hCD59 protein expression was not detected in any tissues we tested in Cre-negative ihCD59 mice or in naive wild-type C56BL/6 mice, but hCD59 protein was highly expressed in all tissues we have tested in Meox2-Cre ${ }^{+}$hCD59 ${ }^{+}$mice (+ represents the hemizygous transgenic genotype). This suggests that hCD59 is constitutively expressed throughout Meox2-Cre ${ }^{+}$ihCD59+ mice.

To determine whether ihCD59 mice can be used to ablate various types of immune cells, ihCD59 transgenic (ihCD59+) mice were crossed with $\mathrm{Lck}-\mathrm{Cre}{ }^{+}, \mathrm{Lysm}-\mathrm{Cre}{ }^{+}$, or $\mathrm{Cd11c}-\mathrm{Cr} \mathrm{e}^{+}$mice to generate several lines of double-transgenic mice. Of note, the lymphocyte protein tyrosine kinase $(L c k)$ and lysozyme $2(L y s m)$ genes are primarily expressed by $\mathrm{T}$ cells and myeloid cells, respectively. Thus, Lck-Cre and Lysm-Cre are widely used Cre lines for deleting floxed sequences in T and myeloid cells, respectively. The Cd11c-
Cre mouse is an often used Cre-lox tool for the deletion of floxed sequences in DCs. Indeed, flow cytometry analysis confirmed specific hCD59 expression in T cells in $\mathrm{Lck}_{-} \mathrm{Cr}^{+}$ihCD59+ mice (Figure 1C), myeloid cells (e.g., monocytes and neutrophils) in Lysm-

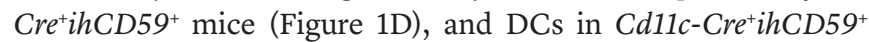
mice (Supplemental Figure 3A). To test whether ihCD59 is functional, splenocytes from $\mathrm{Lck}-\mathrm{Cr} \mathrm{e}^{+} i h \mathrm{CD} 59^{+}$mice were isolated and incubated with ILY in vitro. As illustrated in Figure 1E, incubation with ILY for only 10 minutes lysed almost all of the hCD59+ ${ }^{+}$splenocytes in vitro. Furthermore, Figure $1 \mathrm{~F}$ shows that an in vitro incubation with ILY killed almost all of the T cells without affecting the B cells of $\mathrm{Lck}-\mathrm{Cr} \mathrm{e}^{+} \mathrm{ihCD} 59^{+}$mice.

Next, the in vivo administration of a single dose of ILY mediated a specific and rapid depletion of $\mathrm{T}$ cells $\left(\mathrm{CD} 4^{+}\right.$or $\left.\mathrm{CD} 8^{+}\right)$ and myeloid cells $\left(\mathrm{CD}_{\left.11 \mathrm{~b}^{+}\right)}\right.$in the peripheral blood of $L c k-$ $\mathrm{Cre}^{+} i \mathrm{hCD} 59^{+}$and $\mathrm{Lysm}-\mathrm{Cr} \mathrm{e}^{+} \mathrm{ihCD} 59^{+}$mice, respectively (Figure 2A). As a result, the percentage of the nonablated CD4 and CD8 double-negative cells was relatively increased (Figure 2A). The injection of ILY also rapidly and efficiently depleted DCs and T cells in the spleens of Cd11c-Cre ${ }^{+}$ihCD59 $9^{+}$and $\mathrm{Lck}-\mathrm{Cr} \mathrm{C}^{+} \mathrm{ihCD} 59^{+}$ mice, respectively (Supplemental Figure 3, A and B). This deletion is very specific. For example, the injection of ILY into LckCre ${ }^{+}$ihCD $59^{+}$mice deleted $\mathrm{T}$ cells by approximately $85 \%$ in peripheral blood, but did not affect B cells, neutrophils, monocytes, or NK cells (Figure 2B). This specific deletion of T cells in Lck-Cre ${ }^{+}$ihCD59 $9^{+}$mice is also dose dependent (Figure 2C). ILY administration also specifically deleted $\mathrm{T}$ cells in the spleen, but to a lesser extent (Supplemental Figure 3B). In addition, ihCD59+/- mice were crossed with $\mathrm{Cd} 4-\mathrm{Cre}^{+}$mice to generate Cd4-Cre ${ }^{+}$ihCD59+ mice. The injection of ILY also rapidly depleted $\mathrm{T}$ cells $\left(\mathrm{CD}^{+}\right.$or $\left.\mathrm{CD} 8^{+}\right)$in circulation and in the spleen (data not shown). Of note, although ILY efficiently killed $\mathrm{CD}^{+}$and $\mathrm{CD}^{+} \mathrm{CD}^{+}$thymocytes of $\mathrm{Lck}-\mathrm{Cre} \mathrm{i}^{+} \mathrm{ihCD} 59^{+}$mice in vitro, the in vivo administration of ILY did not affect $\mathrm{CD} 4^{+}$or $\mathrm{CD} 8^{+} \mathrm{T}$ cells in the thymus in these mice (Supplemental Figure 3C). This result also agrees with the finding that the injection of DT efficiently killed T cells in the peripheral blood, but not the thymus of Cd4$\mathrm{Cre}^{+}{ }_{\mathrm{iDTR}}^{+}$mice (1). The resistance of T cells in the thymus to ILYmediated cell ablation was likely due to the insufficient delivery of ILY to the thymus because ILY efficiently lysed thymic T cells in vitro (Supplemental Figure 3C). Interestingly, although ILY did not kill $\mathrm{T}$ cells within the thymus, it triggered increased cell proliferation in the medulla of the thymus (Supplemental Figure 3D) where T cell development occurs.

To test whether increasing amounts of ILY cause more efficient cell ablation in $\mathrm{Lck}_{-} \mathrm{Cr} \mathrm{e}^{+} \mathrm{ihCD} 59^{+}$mice, 150 to $300 \mathrm{ng} / \mathrm{g}$ ILY was injected into the mice. As illustrated in Supplemental Figure 4, injection of high doses of ILY (150-300 ng/g) ablated more than $95 \%$ of circulating $\mathrm{T}$ cells in Cd4-Cre $e^{+} i h C D 59$ mice, but injection of such saturated doses of ILY only ablated approximately $40 \%$ of $\mathrm{T}$ cells in the spleen and did not affect $\mathrm{T}$ cells in the thymus. This partial or resistant ablation was probably due to a consequence of impaired local delivery of ILY because ILY was equally efficient in lysing more than $95 \%$ of peripheral, thymic, or splenic T cells in vitro (Supplemental Figure 3). Consistently, we did not observe any detectable off-target effects or toxicities on nontargeted organs examined in this $\mathrm{T}$ cell-ablation model 
A
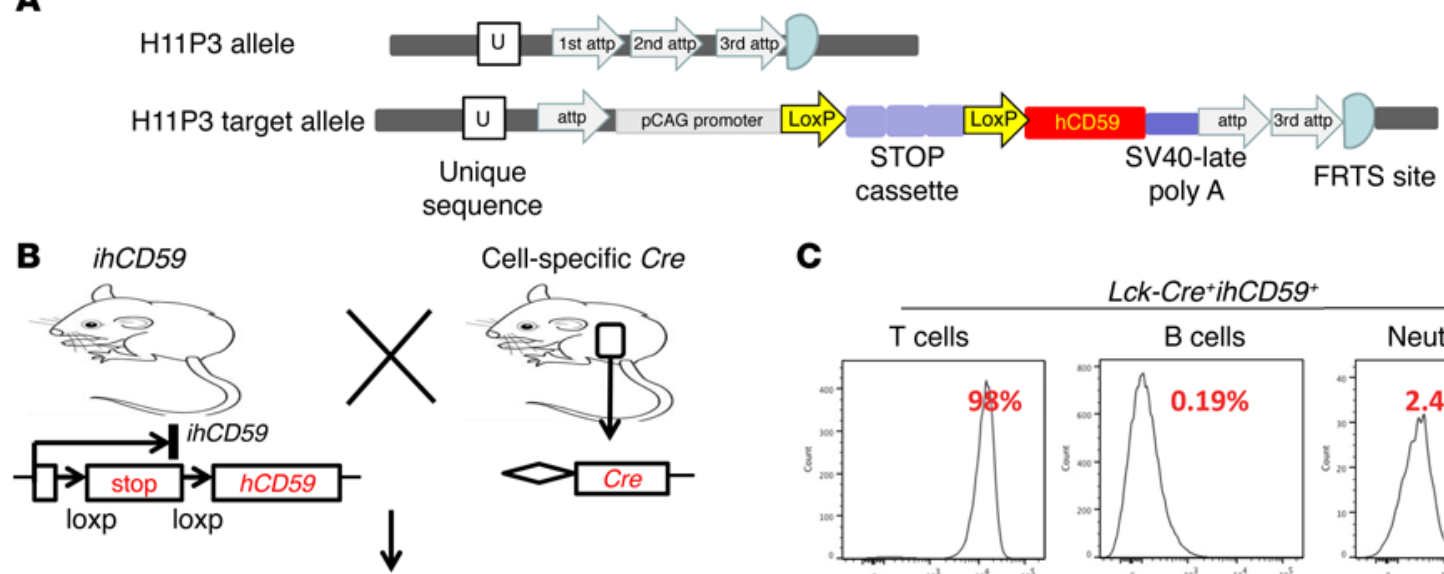

Cell-specific Cre

C
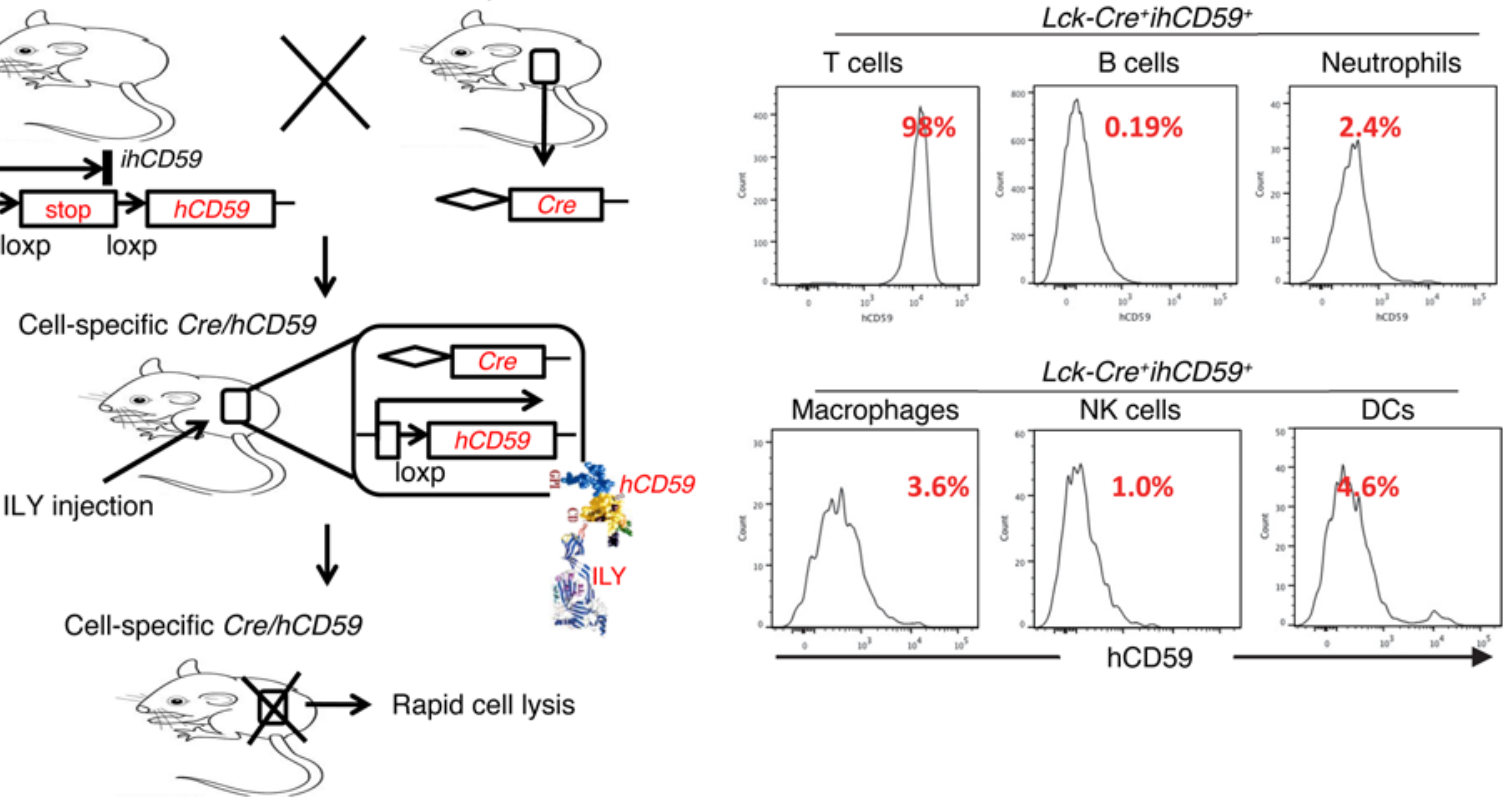

D

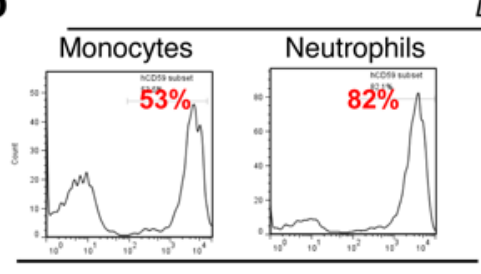

Lysm-Cre+ihCD59+

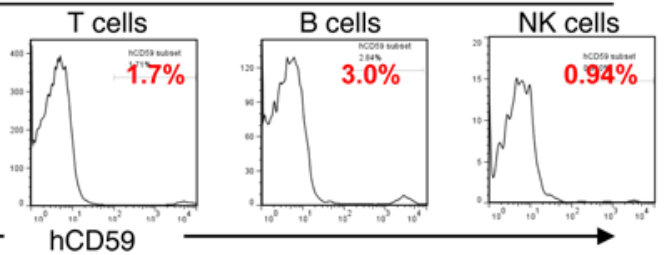

$\mathbf{E}$
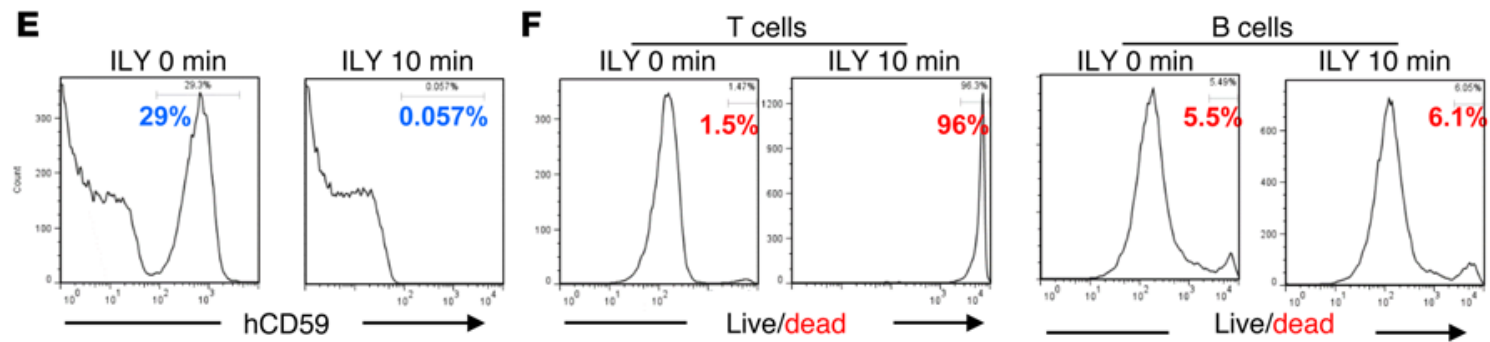

Figure 1. Generation of ihCD59 knockin mice. (A) Map of the pBT378-CAG-LSL-hCD59 vector for pronuclear injection. (B) General strategies for the generation of ihCD59 mice. The STOP cassette, which prohibits transgene expression, is removed by crossing the inducible transgenic strain to a cell-specific Creexpressing mouse strain. The consequent expression of the transgene renders the respective tissues sensitive to rapid cell lysis induced by the injection of ILY. (C and D) Representative FACS analyses of hCD59 expression on T cells in Lck-Cre+ihCD59+ mice (C) and on monocytes/neutrophils in Lysm-Cre ${ }^{+}$ihCD59 ${ }^{+}$ mice (D). (E and F) Splenocytes that were isolated from $L c k-C r e^{+} i h C D 59^{+}$mice were incubated with ILY in vitro for 10 minutes. FACS analyses were performed. E shows $29 \%$ live hCD59+ spleen cells and $0.057 \%$ live hCD59+ spleen cells before and after ILY incubation, respectively. F shows live/dead T and B cells. In $\mathbf{C}$ and $\mathbf{D}$, the representative graphs from 5 mice are shown. In $\mathbf{E}$ and $\mathbf{F}$, the representative graphs from 4 mice are shown.

injected with the saturated doses of ILY except for the elevated serum lactate dehydrogenase levels, which likely reflect the death of T cells (Supplemental Figure 5).

The dynamic recovery of each immune cell population in circulation and in tissues after the rapid ablation with a single ILY injection was examined. As illustrated in Figure 2, D and E, after a single ILY injection, circulating T cells in $L c k-C r e^{+} i h C D 59^{+}$mice and circulating monocytes in $\mathrm{Lysm}$-Cre ${ }^{+} \mathrm{ihCD} 59^{+}$mice were rapidly depleted by approximately $90 \%$ within 0.1 hours, but recovered to near normal levels 24 hours after injection. Interestingly, in ILY-treated $\mathrm{Lysm}-\mathrm{Cre}^{+} \mathrm{ih} C \mathrm{CD} 59^{+}$mice, $\mathrm{Ly} 6 \mathrm{C}^{-}$monocytes recovered much more slowly than Ly6 $\mathrm{C}^{+}$monocytes (Figure 2E). In addition, the injection of ILY also efficiently depleted T cells and DCs in the spleens of $\mathrm{Lck}-\mathrm{Cr} \mathrm{e}^{+} i h \mathrm{CD} 59^{+}$and $\mathrm{Cd} 11 \mathrm{c}-\mathrm{Cre} \mathrm{e}^{+} i h \mathrm{CD} 59^{+}$ 

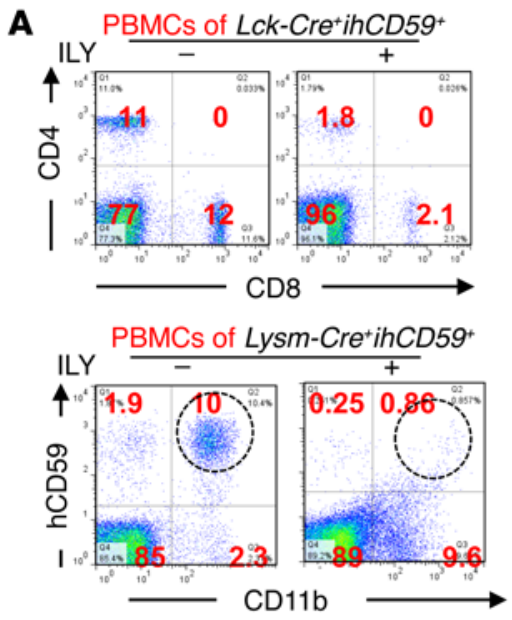
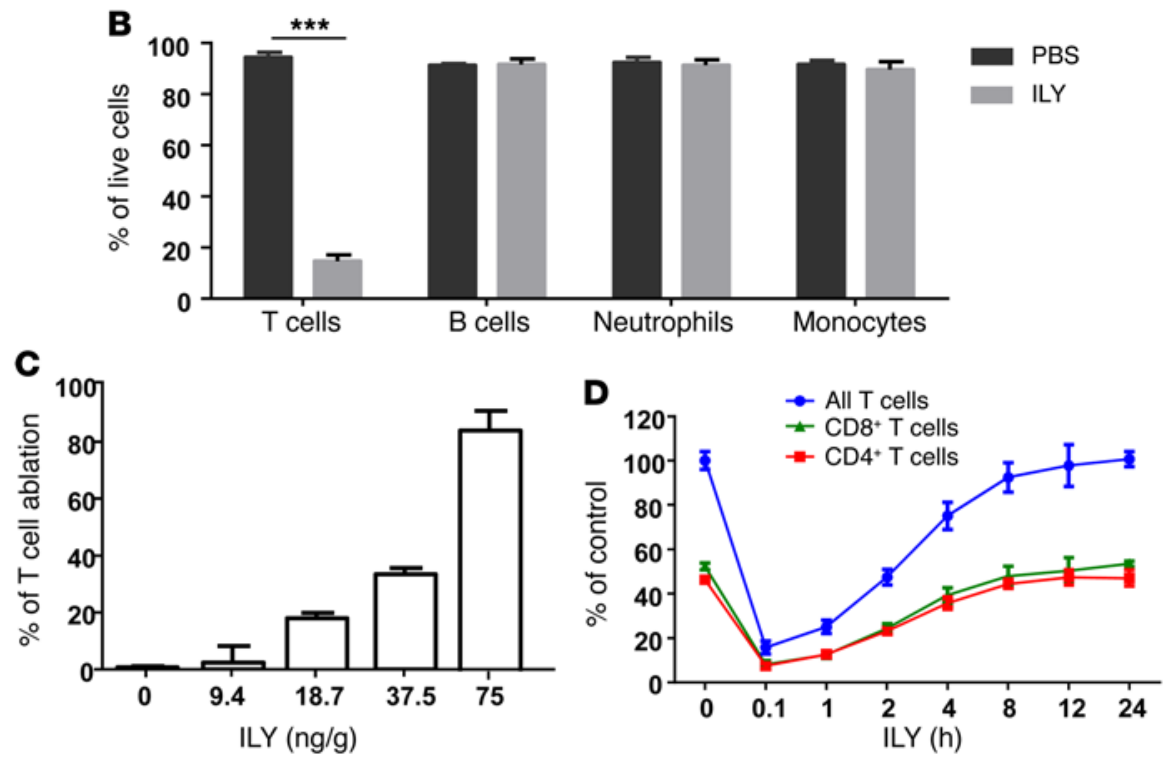
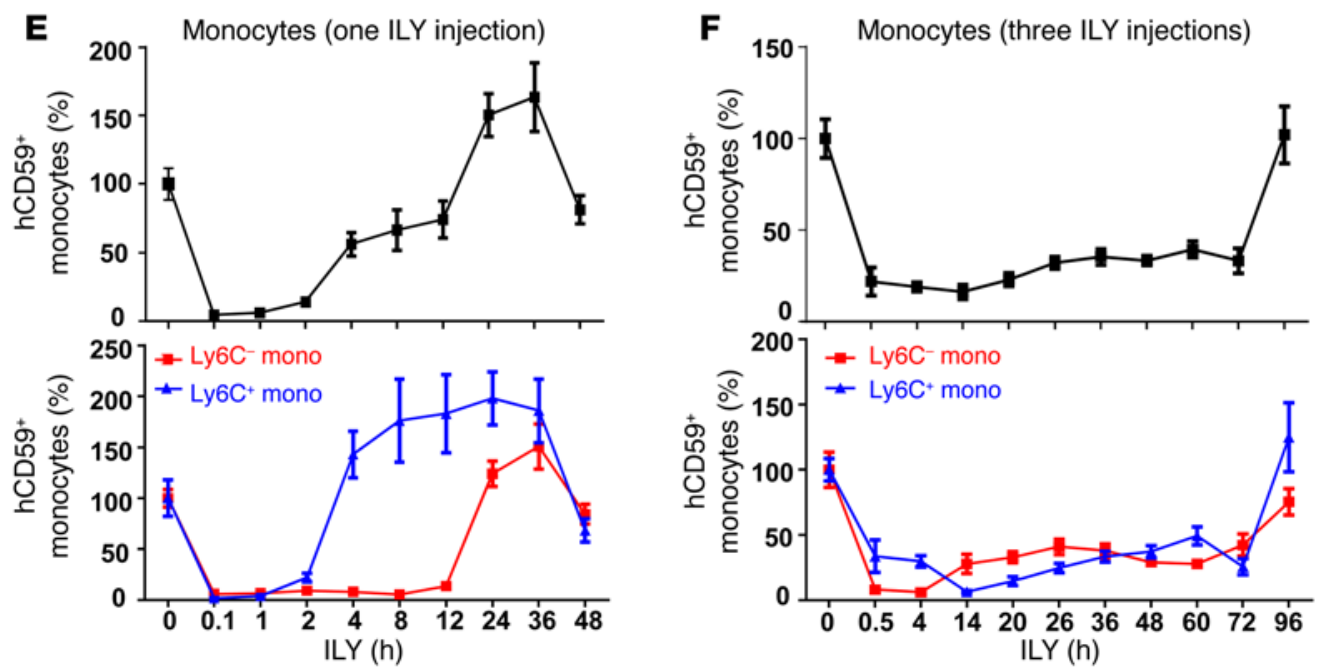

Figure 2. ILY treatment rapidly and specifically induces immune cell ablation in vivo. (A and B) Representative FACS analyses of PBMCs from 2 lines of double-transgenic mice that were treated with or without ILY (75 ng/g, i.v., $n=4)$. A shows the ablation of T cells $\left(C D 4^{+}\right.$or CD8 $\left.{ }^{+}\right)$and CD11b ${ }^{+}$myeloid cells. B shows the percentage of live cells. (C) Cd4-Cre+ihCD59+ mice were treated with different doses of ILY for 10 minutes, and the efficiency of T cell ablation was determined by FACS and summarized $(n=4)$. (D) Dynamic changes in the percentage of total T, CD4 ${ }^{+}$, and CD8 ${ }^{+}$T cells in PBMCs after a single ILY injection $(75 \mathrm{ng} / \mathrm{g}$, i.v., $n=4)$. (E and $\mathbf{F})$ Dynamic changes in the percentage of circulating hCD59+, Ly6C-', and Ly6C+ monocytes in Lysm-Cre+ $i h C D 59^{+}$after $1(\mathbf{E})$ or multiple (F) ILY injections ( $n=3$ for 0 hours and $n=5$ for all other time points). The percentage of monocytes was set at $100 \%$ at the 0 -hour time point (before ILY treatment). The values in $\mathbf{B}-\mathbf{F}$ represent mean \pm SD. ${ }^{* *} P \leq 0.001$, as determined by 2 -tailed Student's $t$ test.

mice, respectively. The recovery to near normal levels occurred 24 hours after injection (Supplemental Figure 6, A and B).

To obtain a more prolonged ablation, $\mathrm{Lysm}-\mathrm{Cre}{ }^{+} i h \mathrm{CD} 59^{+}$mice were treated with multiple ILY injections (3 injections once a day). As illustrated in Figure 2F, the monocytes were reduced by $80 \%$ to $90 \%$ within 0.5 hours after the first injection, and this reduction was maintained for 3 days of ILY injection. After ILY injection was stopped, monocytes started to recover and returned to normal levels. This result suggests that multiple ILY injections are able to induce the chronic ablation of immune cells.

In the liver, there are 2 populations of macrophages: Kupffer cells (liver-resident macrophages, $\mathrm{F} 4 / 80^{\text {hi }}$ ) and infiltrating macrophages $\left(\mathrm{F} 4 / 80^{\mathrm{lo}}\right)(13)$. To determine whether ILY efficiently depletes liver macrophages, immunostaining with $\mathrm{F} 4 / 80$ was performed. As illustrated in Figure 3A, approximately $60 \%$ of the $\mathrm{F} 4 / 80^{+}$cells from $\mathrm{Lysm}-\mathrm{Cr} \mathrm{e}^{+} \mathrm{ihCD} 59^{+}$mice coexpressed hCD59, as demonstrated by the colocalization of the macrophage/ Kupffer cell-specific marker F4/80 with hCD59. ILY injection into $\mathrm{Lysm}$-Cre $\mathrm{ihCD}^{+} 9^{+}$mice markedly reduced the number of $\mathrm{F} 4 / 80^{+}$cells as early as 4 hours after injection; these reached the lowest level at 12 hours and recovered at 36 hours (Figure 3, $\mathrm{B}$ and $\mathrm{C})$. Using a BrdU incorporation assay demonstrated that the peak time of $\mathrm{F} 4 / 80^{+}$Kupffer cell proliferation occurred 36 hours after ILY injection (Figure 3, B and C), suggesting that the proliferation of Kupffer cells contributes to the replenishment of Kupffer cells after depletion.

To determine whether monocytes also partly contribute to Kupffer cell replenishment, we utilized the adoptive transfer of 
A
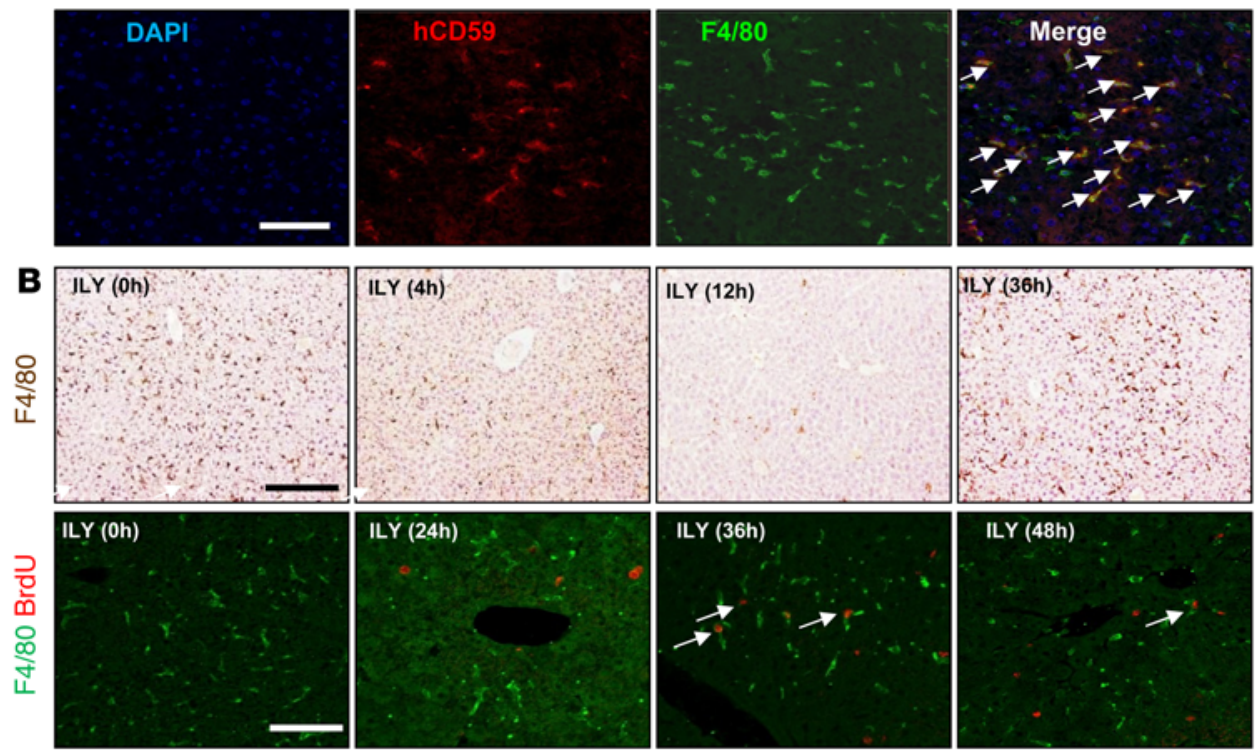

D

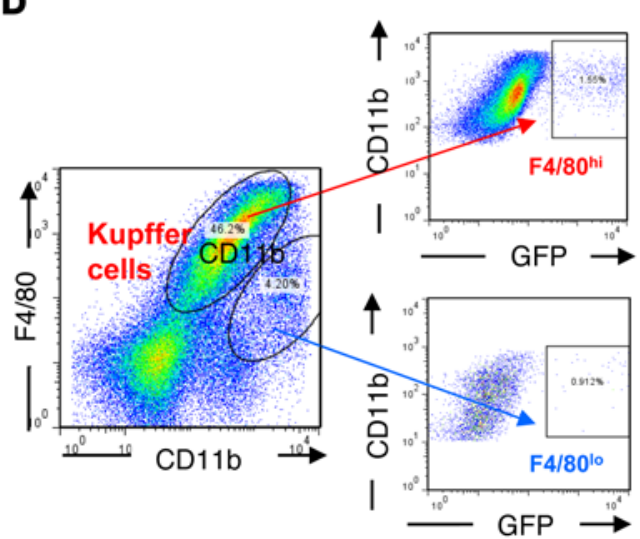

C
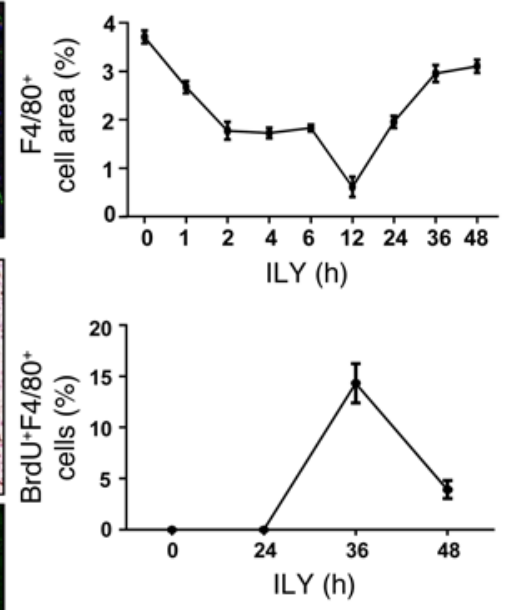

Figure 3. ILY treatment depletes liver Kupffer cells in $\mathbf{L y s m - C r e +}{ }^{+}$ihCD59+ mice: a model to study Kupffer cell repopulation. (A) Representative immunofluorescence staining of hCD59 and F4/80 in livers from 4 different Lysm-Cre+ihCD59+ mice. Arrows indicate the coexpression of F4/80 (green) and hCD59 (red) in the Kupffer cells. (B) Representative F4/80 immunostaining and double immunofluorescence with F4/80 and BrdU in livers from 3 different Lysm-Cre+ihCD59+ mice that were treated with ILY (75 ng/g, i.v.). BrdU (50 mg/kg) was given 2 hours before sacrifice, and mice were euthanized at various

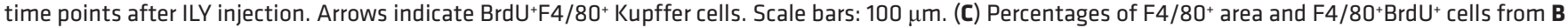
were quantified. Values represent mean $\pm \mathrm{SD}$ ( $n=3$ for 0 hours and $n=6$ for all other time points). (D) A total of $7.5 \times 10^{6} \mathrm{GFP}^{+}$monocytes from ROSA26EGFP transgenic mice were adoptively transferred into ILY-treated Lysm-Cre+ihCD59+ mice in which the Kupffer cells were depleted. Three days later, liver macrophages were isolated and subjected to FACS analyses. Kupffer cell-like GFP+CD11b ${ }^{\text {lo }}$ F4/80 $/ 8$ cells were identified $(n=4)$.

$\mathrm{GFP}^{+}$monocytes to mice. As illustrated in Supplemental Figure 6C, few Kupffer cell-like $\mathrm{GFP}^{+} \mathrm{CD} 11 \mathrm{~b}^{\mathrm{lo}} \mathrm{F} 4 / 80^{\mathrm{hi}}$ cells were identified after adoptive transfer of $\mathrm{GFP}^{+}$monocytes into wild-type C57BL/6 mice. The lack of conversion of monocytes to Kupffer cell-like cells may be either because monocytes do not contribute to Kupffer cells or because normal mouse livers contain a large number of Kupffer cells that prevent monocyte conversion. To answer these questions, $\mathrm{GFP}^{+}$monocytes were adoptively transferred to ILY-treated $\mathrm{Lysm}$-Cre ${ }^{+} i h C D 59^{+}$mice in which Kupffer cells were depleted. Interestingly, approximately $1.5 \%$ of $\mathrm{F} 4 / 80^{\text {hi }} \mathrm{CD} 11 \mathrm{~b}^{\text {lo }}$ Kupffer cells were $\mathrm{GFP}^{+}$cells that were contributed from $7.5 \times 10^{6}$ transferred $\mathrm{GFP}^{+}$monocytes (Figure 3D). These results suggest that monocytes can be converted into Kupffer cells after acute cell loss, highlighting that the ihCD59 model is useful for studying immune cell differentiation and repopulation.
Application of ihCD59 mice for dissecting cellular functionalities in immune-mediated acute diseases. To assess whether the ihCD59 model can be used to study the roles of lymphocytes in the pathogenesis of immune-mediated acute diseases, we used 2 models of acute hepatitis. First, we used a model of $\alpha$-GalCer-induced NKTmediated acute liver injury to evaluate whether DC ablation by ILY affects antigen presentation to NKT cells. $\alpha$-GalCer is a lipid antigen that activates NKT cells to produce IFN- $\gamma$ and IL-4 via presentation by DCs and results in acute liver injury (14). As illustrated in Figure 4A, the administration of $\alpha$-GalCer markedly elevated serum IFN- $\gamma$ and IL-4 levels as well as serum alanine aminotransferase (ALT) (a marker for liver injury) in PBS-treated ihCD59+ and $\mathrm{Cd} 11 \mathrm{c}-\mathrm{Cr} \mathrm{C}^{+} i \mathrm{C} \mathrm{C} 59^{+}$mice. ILY treatment significantly blocked $\alpha$-GalCer-induced cytokine production and serum ALT elevation in $\mathrm{Cd} 11 \mathrm{c}-\mathrm{Cr} \mathrm{e}^{+} \mathrm{ihCD} 59^{+}$but not in $\mathrm{ihCD} 59^{+}$mice. 

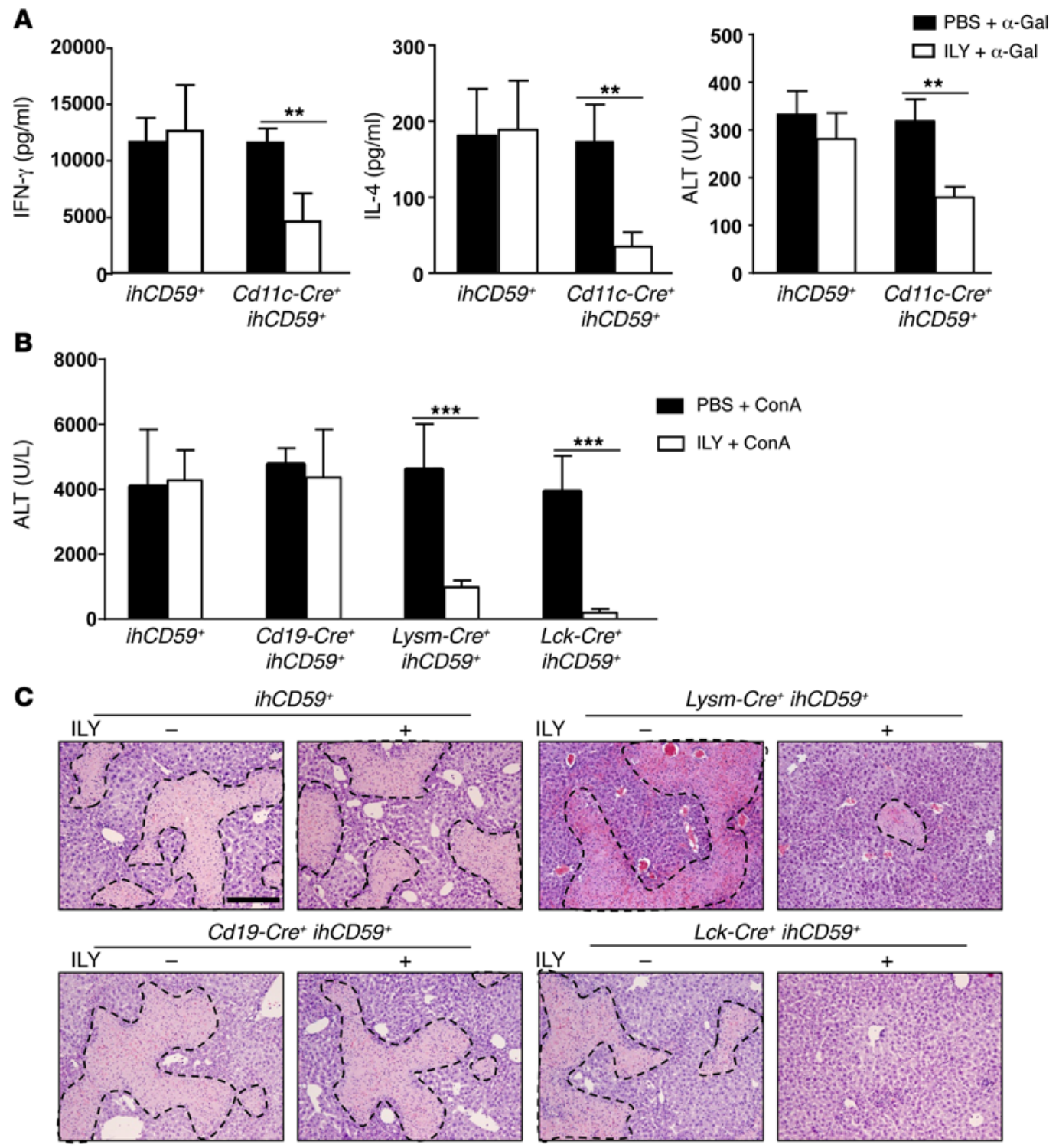

Figure 4. Application of the ihCD59 model in the study of acute immune-mediated hepatitis. (A) $C d 11 c-C r e^{+} i h C D 59^{+}$mice were given a single PBS or ILY (75 ng/g, i.v.) injection 1 hour before the injection of $100 \mu \mathrm{g} / \mathrm{kg}$ (i.v.) $\alpha$-GalCer ( $\alpha$-Gal). The serum cytokines and ALT were determined 24 hours after $\alpha$-GalCer injection $(n=4)$. (B and C) Effects of B cells, T cells, and myeloid cells on Con A-induced acute hepatitis. Several lines of Cre ${ }^{+}$ihCD59 ${ }^{+}$mice were given a single PBS or ILY (75 ng/g, i.v.) injection 1 hour before the injection of $12 \mathrm{mg} / \mathrm{kg}$ (i.v.) Con A. Mice were sacrificed 24 hours after Con A injection. Serum ALT levels $(\mathbf{B})$ and H\&E staining $(\mathbf{C})$ were examined $(n=8)$. Scale bar: $100 \mu \mathrm{m}$. Dashed lines indicate necrotic areas. Values represent mean \pm SD. ${ }^{* *} P<0.01$; ${ }^{* *} P<0.001$, as determined by 2-tailed Student's $t$ test.

Second, we performed concanavalin A-induced (Con Ainduced) acute hepatitis, a widely used model for studying immune-mediated liver injury (15). In this model, several types of immune cells have been implicated; however, to what degree these immune cells contribute to Con A-induced hepatitis remains unclear. To address this question, T, B, and myeloid cells were depleted in ILY-treated $\mathrm{Lck}-\mathrm{Cr} \mathrm{e}^{+} \mathrm{ihCD} 5 \mathrm{9}^{+}$, CD19-Cre ${ }^{+} i h C D 59^{+}$, and $\mathrm{Lysm}-\mathrm{Cre} \mathrm{C}^{+} i h \mathrm{CD} 59^{+}$mice, respectively, followed by Con A injection. As illustrated in Figure 4, B and $\mathrm{C}$, the injection of Con A alone without ILY injection induced marked liver injury, as demonstrated by the elevation of serum ALT and massive liver necrosis in all of the strains. Pretreatment with ILY completely abolished Con A-induced liver injury in $\mathrm{Lck}-\mathrm{Cr} \mathrm{e}^{+} \mathrm{ihCD} 59^{+}$mice and ameliorated liver injury to a lesser extent in $\mathrm{Lysm}-\mathrm{Cre}^{+} \mathrm{ihCD} 59^{+}$mice, but did not affect liver injury in $C D 19-C r e^{+} i h C D 59^{+}$or $i h C D 59^{+}$mice. These results suggest that both $\mathrm{T}$ and myeloid cells play essential roles, while $\mathrm{B}$ cells are not involved in promoting Con A-induced liver injury.

Application of ihCD59 for dissecting the cellular functionalities in the pathogenesis of chronic immune-mediated diseases. To evaluate whether the ihCD59 model is useful for dissecting the cellular functionality in the pathogenesis of chronic diseases, we used an experimental autoimmune encephalomyelitis (EAE) rodent model that is widely used for multiple sclerosis research (16). Although the roles of various types of immune cells have previously been investigated in the EAE model with different methodologies, there has been no comparison of the contribution of these cells to EAE pathogenesis. To make this comparison, 3 lines of $\mathrm{Cre}^{+} h \mathrm{CD} 59^{+}$ 
A

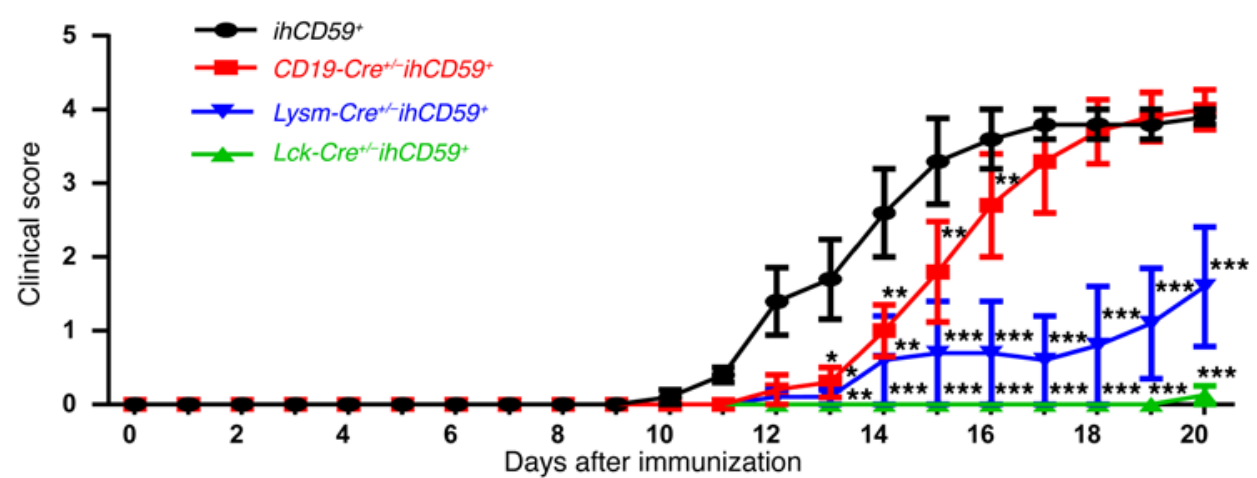

B
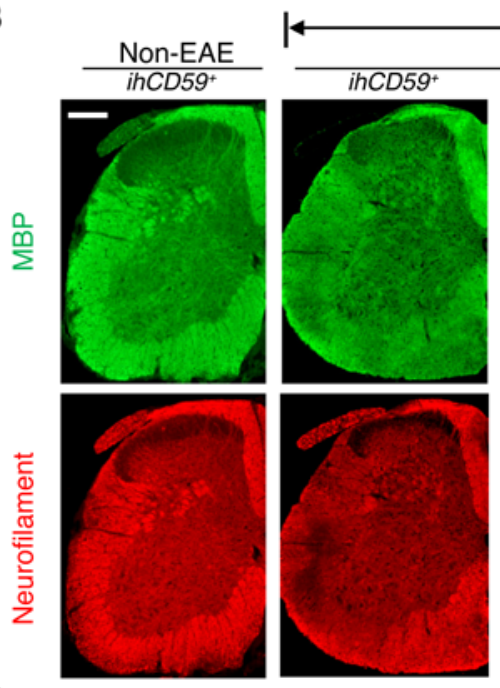

ILY treatment
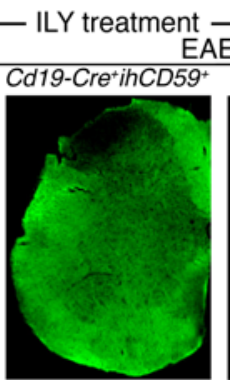

\section{EAE}
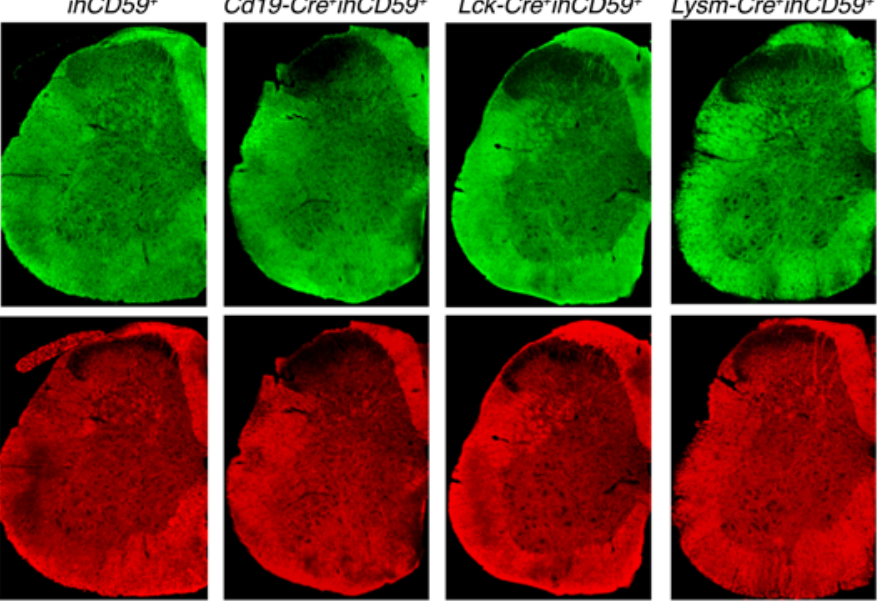

C
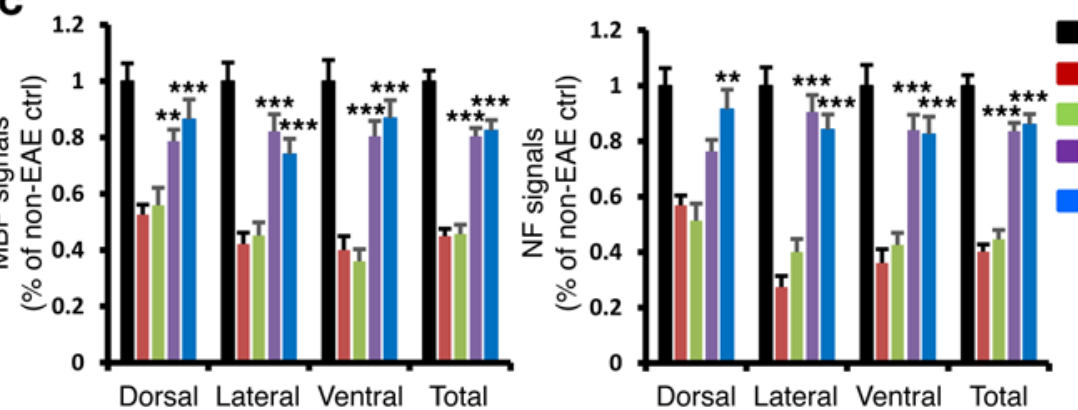

Figure 5. Application of the ihCD59 model in the study of chronic EAE pathogenesis. Several lines of $\mathrm{Cre}^{+} \mathrm{ihCD} \mathrm{Cg}^{+}$ mice were given MOC immunization; 3 days later, ILY was injected (i.p., $100 \mathrm{ng} / \mathrm{g}$, daily) for 14 days, and mice were euthanized 20 days after MOG immunization. (A) Clinical EAE scores $(n=8)$. ${ }^{*} P<0.05 ;{ }^{*} P<0.01 ;{ }^{* * *} P<$ 0.001 (vs. ihCD59+ group, as determined by 2-way ANOVA followed by Bonferroni's post-hoc test). (B) Analysis of transverse sections of the spinal cord by immunostaining for MBP (upper panel) and neurofilament (lower panel). Representative images from 8 mice are shown. In all of the sections, dorsal is up. Scale bar: 250 $\mu \mathrm{m}$. (C) The levels of myelin signals as stained by MBP and axonal signals as labeled by neurofilament (NF) in different white matter areas of transverse sections from various groups of mice were determined $(n=8)$. Values represent mean \pm SD. ${ }^{*} P<0.01 ;{ }^{* *} P<0.001$ (vs. EAE ihCD59+ as indicated in the figure were determined by 1-way ANOVA followed by Dunnett's post-hoc test). mice were subjected to ILY and myelin oligodendrocyte glycoprotein (MOG) peptide treatment, followed by monitoring of the clinical EAE score. As illustrated in Figure 5A, the ablation of T cells in $\mathrm{Lck}-\mathrm{Cre}^{+} h \mathrm{CD} 59^{+}$mice completely prevented EAE development, and the ablation of myeloid cells in $\mathrm{Lysm}-\mathrm{Cre}^{+} h \mathrm{CD} 59^{+}$mice also attenuated the EAE scores, but to a lesser extent. In contrast, the EAE scores were only modestly reduced in the B cell-ablated $\mathrm{CD} 19-\mathrm{Cre}^{+} i h \mathrm{CD} \mathrm{9}^{+}$mice. Histological analyses revealed that the ablation of $\mathrm{T}$ cells or myeloid cells but not $\mathrm{B}$ cells enhanced myelination, as demonstrated by myelin basic protein (MBP) staining, and increased the axon number, as demonstrated by neurofilament staining (Figure 5, B and C). These 2 methods are used to measure myelination and axon integrity in the lumbar spinal cord in EAE mice (17). These findings suggest that chronic ILY treatment can be used to study the functions of different immune cells in chronic immune-mediated diseases, such as EAE.
Highly efficient and selective ablation of epithelial cells: models for organ damage and regeneration. To further address whether the ihCD59 model can be used to ablate epithelial cells in solid organs, we developed several models that selectively ablated epithelial cells in the liver. Of note, the liver has 2 types of epithelial cells, including hepatocytes and biliary epithelial cells (BECs), both of which are derived from hepatoblasts that express albumin (Alb). Therefore, the Alb-Cre mouse is a widely used Cre line to delete a floxed sequence in both hepatocytes and BECs (18). In $\mathrm{Alb}-\mathrm{Cr} e^{+} \mathrm{CD} 59^{+}$ mice, hCD59 protein expression was specifically detected in both hepatocytes and BECs in the liver (Figure 6A). ILY administration to $\mathrm{Alb}$-Cre $\mathrm{ihCD}^{+} \mathrm{9}^{+}$mice rapidly induced a dramatic elevation of serum ALT levels (indication of hepatocyte injury) and elevation of bilirubin levels (indication of bile duct injury) (Figure 6A). Importantly, ILY injection induced liver damage (elevation of serum ALT and aspartate aminotransferase [AST]) in a dose-dependent 
A

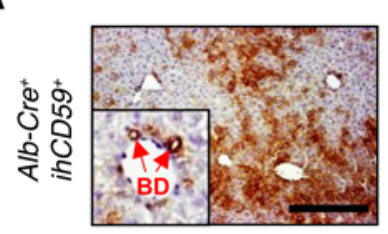

B

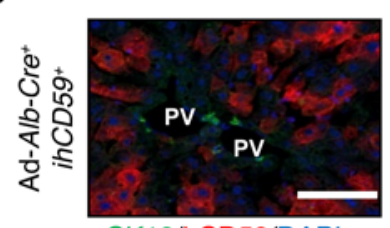

C

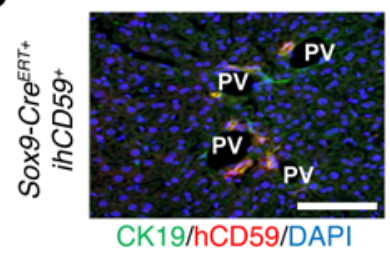

D
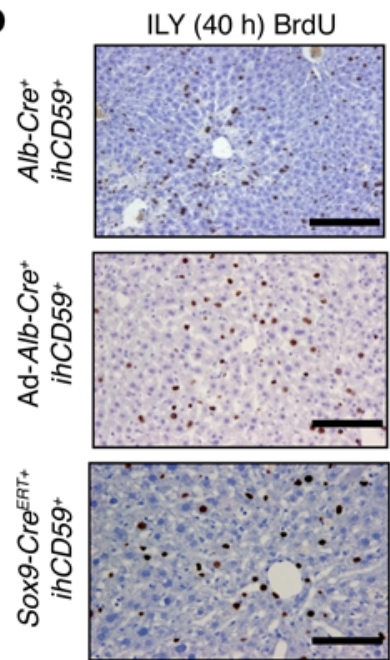
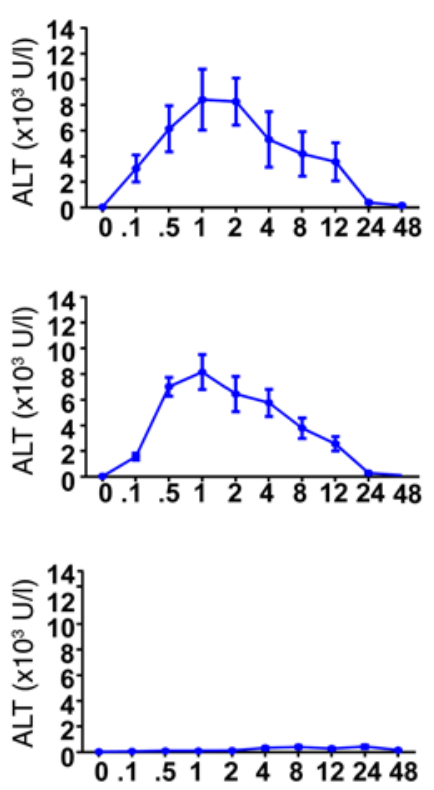

E
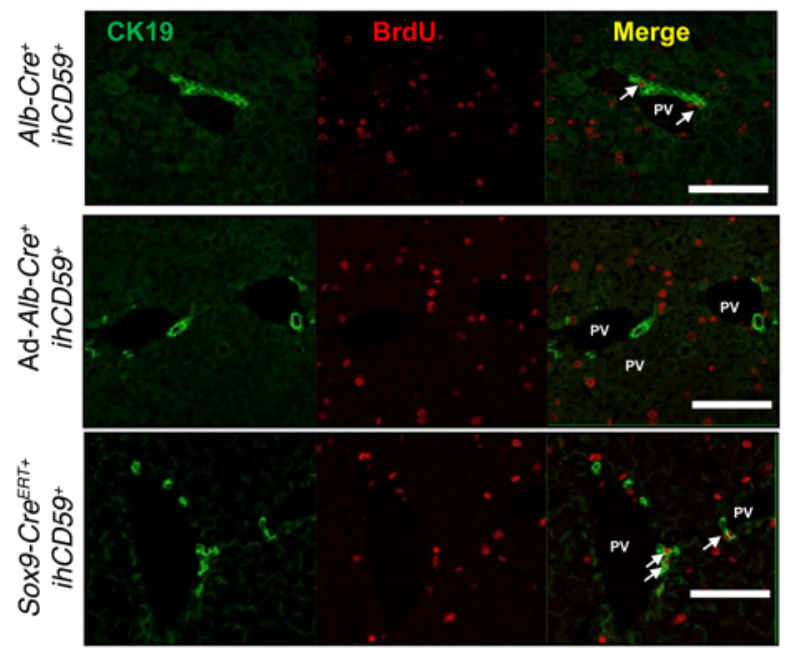

$\mathbf{F}$

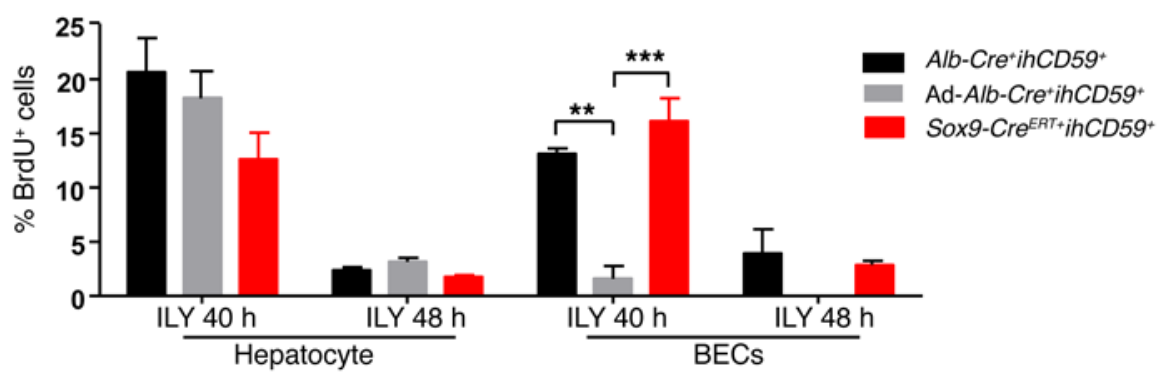

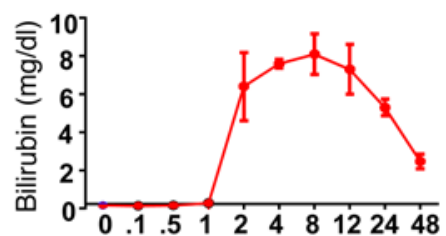
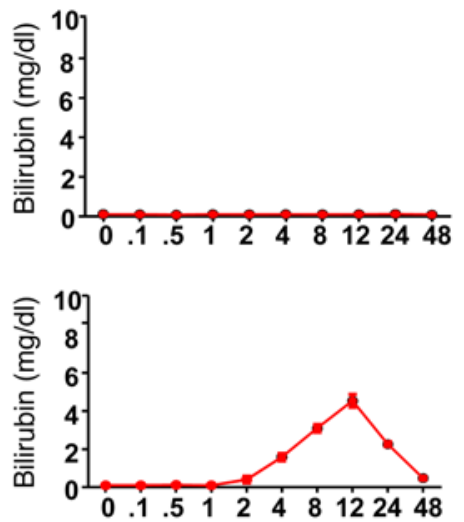

PV hours after ILY treatment were determined (F). Scale bars: 100 $\mu \mathrm{m}$. Values represent mean \pm SD $(n=9) .{ }^{*} P<0.01 ;{ }^{* *} P<0.001$. Comparisons between groups as indicated in the figure were determined by 1-way ANOVA followed by Bonferroni's posthoc test. manner, with the highest peak levels of ALT (approximately 9,000 IU/l) after the injection of $150 \mathrm{ng} / \mathrm{g}$ ILY, followed by approximately 3,000 and 1,000 IU/1 ALT after the injection of $75 \mathrm{ng} / \mathrm{g}$ and 37.5 ng/g ILY, respectively (Supplemental Figure 7A). H\&E staining showed that the injection of 150,75 , and $37.5 \mathrm{ng} / \mathrm{g}$ ILY induced necrosis in approximately $30 \%, 10 \%$, and $2 \%$ of the liver area of Alb-Cre ${ }^{+}$ihCD59 $9^{+}$mice, respectively (Supplemental Figure 7B). Moreover, H\&E staining confirmed the damage to hepatocytes (necrosis) and bile duct destruction (Supplemental Figure 7, B and C), and CK19 (specific for BECs) staining further confirmed the bile duct destruction (Supplemental Figure 7D) in ILY-treated Alb$C r e^{+} i h C D 59^{+}$mice. In contrast, ILY treatment had no effect on liver injury in ihCD59+ mice (Supplemental Figure 7, A and B).

Second, to exclusively express hCD59 in hepatocytes, we infected $i h C D 59^{+}$mice with an adenovirus-expressing Cre that is controlled by the Alb promoter (Ad-Alb-Cre) to develop a model in which hCD59 is expressed only in hepatocytes through a viral delivery system (Ad-Alb-Cre $\left.{ }^{+} i h C D 59^{+}\right)$. The Ad-Alb-Cre virus induced the expression of the hCD59 protein in hepatocytes (Figure 6B), but not in $\mathrm{CK} 19^{+} \mathrm{BECs}$, desmin ${ }^{+}$hepatic stellate cells, or 
A

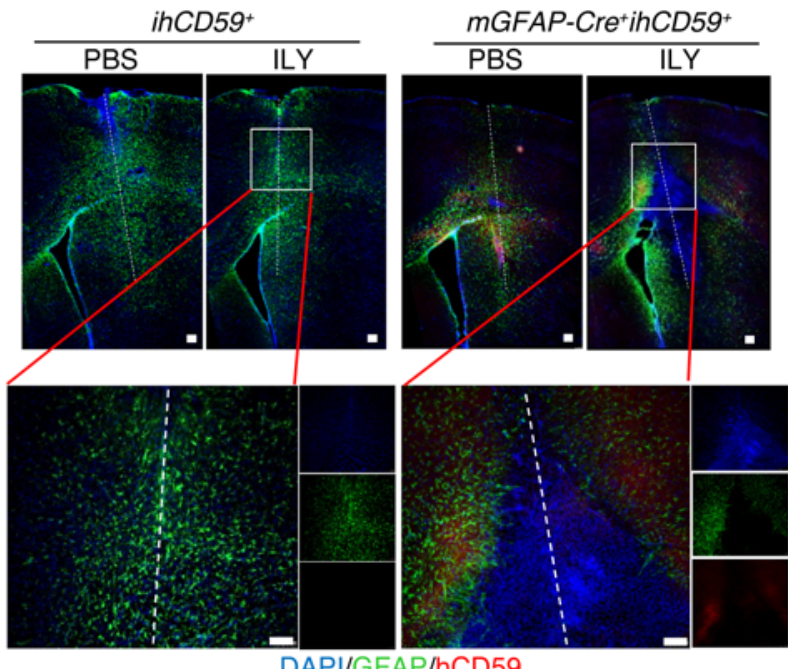

B
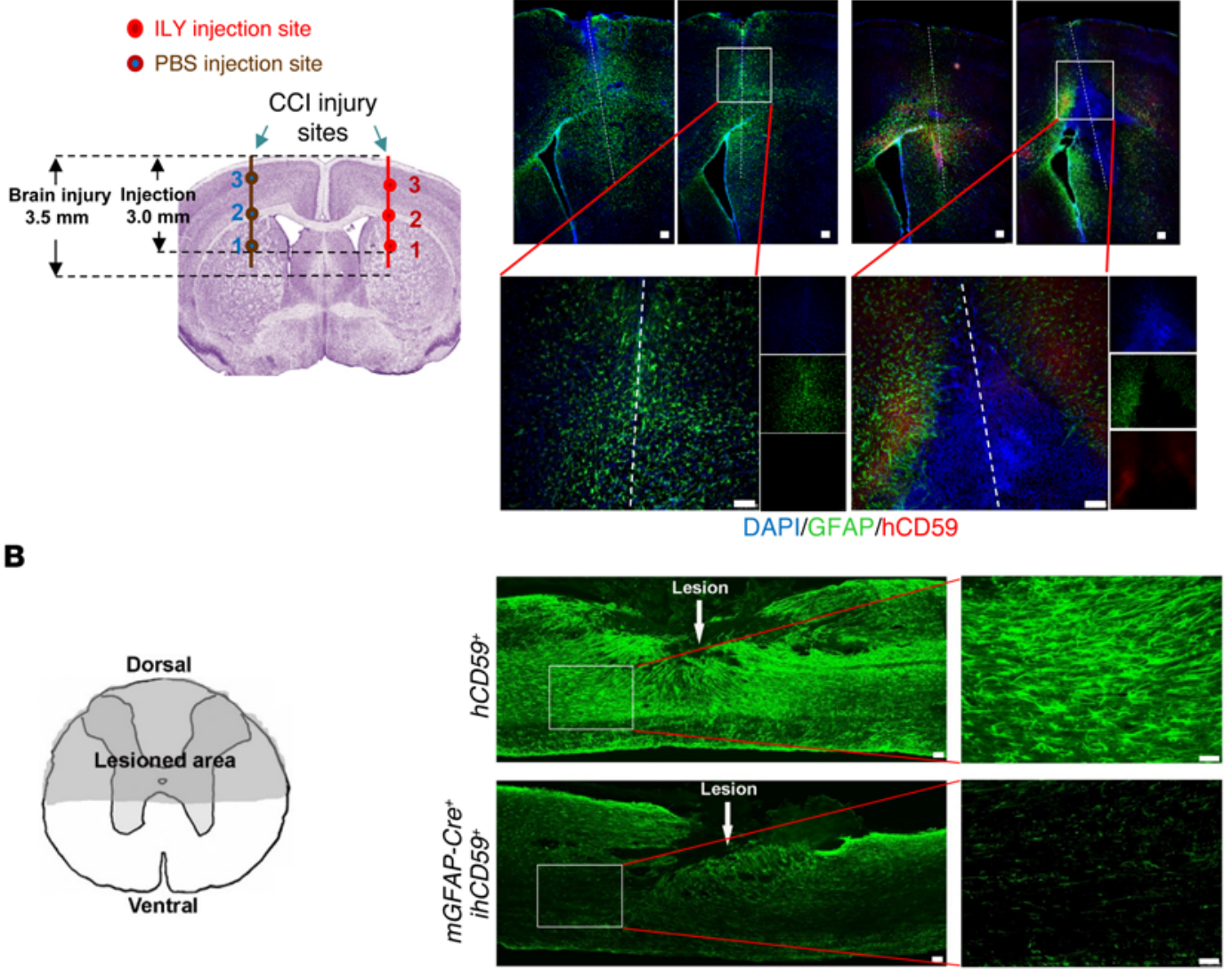

Figure 7. ILY specifically ablates reactive astrocytes. (A) Brain injury model. Left panel indicates the sites in the brain injury model with the local injection of ILY (10 ng/g) or PBS. Left panel adapted with permission from Elsevier Academic Press (32). The costaining of hCD59 (red) and GFAP (green) within the sections of the brain lesion sites is indicated by the white dashed line. The lower pictures are the magnifications of the white box area in the upper image. (B) Spinal cord injury model. The left panel indicates the transected area in the spinal cord at T7 with the local injection of ILY. The area in gray indicates the injury location. Parasagittal sections of the spinal cord around the lesion from a representative mouse in each group (right color images) indicate a high density of GFAP+-reactive astrocytes and scar tissue at/around the lesion site. The images on the right side indicate reactive astrocytes at a higher magnification. Representative images from 5 different mice are shown. Scale bars: $100 \mu \mathrm{m}$.

F4/80+ Kupffer cells (Supplemental Figure 7E). The injection of ILY rapidly induced damage to hepatocytes but not damage to BECs, as demonstrated by the elevation of serum ALT but not bilirubin (Figure 6B). H\&E and CD19 staining also confirmed no bile duct destruction in ILY-treated Ad-Alb-Cre ${ }^{+} i h C D 59^{+}$mice (Supplemental Figure 7, C and D).

Finally, to exclusively express hCD59 in BECs, ihCD59+ mice were crossed with tamoxifen-inducible Sox9-Cre (Sox9-Cre ${ }^{E R T}$ ) mice, a model that is used to express Cre in BECs only in the liver (19). The treatment of Sox9-Cre ${ }^{E R T+} i h C D 59^{+}$mice with tamoxifen induced temporal and specific expression of hCD59 on BECs, but not on hepatocytes, as demonstrated by the double staining of hCD59 and Sox9 (Figure 6C). The injection of ILY into Sox9$\mathrm{Cr} e^{E R T+} i h C D 59^{+}$mice rapidly induced severe bile duct damage, but little hepatocyte damage, as demonstrated by the marked elevation of serum bilirubin, but not ALT (Figure 6C). H\&E and CD19 staining also confirmed bile duct destruction in ILY-treated Sox9$C r e^{E R T+}$ ihCD59 $9^{+}$mice (Supplemental Figure 7, C and D).

Collectively, the above results indicate that the ihCD59 model has high specificity and potency to ablate epithelial cells (e.g., hepatocytes and BECs in the liver), which prompted us to utilize these models to study liver regeneration and BEC regeneration. As illustrated in Figure 6, D-F, significant proliferation of both hepatocytes and BECs was observed in $\mathrm{Alb}-\mathrm{Cr} \mathrm{e}^{+} \mathrm{ihCD} 59^{+}$mice, with the peak effect occurring 40 hours after ILY injection. In contrast, only hepatocyte proliferation and not BEC proliferation was observed in ILY-treated Ad-Alb-Cre ${ }^{+} i h C D 59^{+}$mice. Interestingly, both hepatocyte and BEC proliferation were observed in ILY-treated Sox9-Cre ${ }^{E R T+}$ ihCD59+ mice, despite only BECs being damaged in this model. Thus, these highly selective ablation models can be used to study hepatocyte and/or BEC regeneration.

Multiple injections of ILY to Alb-Cre+ihCD59+ mice induce liver fibrosis and the emergence of liver progenitor cells. To examine chronic liver injury, multiple ILY injections (150 ng/g every 3 days for 9 days) were applied to $\mathrm{Alb}-\mathrm{Cr} e^{+} i h C D 59^{+}, \mathrm{Ad}-\mathrm{Alb}-\mathrm{Cr} \mathrm{C}^{+} i \mathrm{CCD} 59^{+}$, or Sox9-Cre ${ }^{E R T+} i h C D 59^{+}$mice. Mice were sacrificed 48 hours after the last injection. As illustrated in Supplemental Figure 8A, H\&E staining showed a distorted hepatic architectural pattern and chronic liver injury. In addition, significant $\alpha$-smooth muscle actin-positive ( $\alpha$-SMA-positive) staining was observed in the portal vein area, suggesting moderate liver fibrosis after multiple ILY injections. Interestingly, H\&E staining revealed some oval cells (also 
called liver progenitor cells [LPCs]) in the $\alpha$-SMA-positive areas. The presence of LPCs was further confirmed by CK19 staining (Supplemental Figure 8A) and immunofluorescence staining with Sox9 and EpCAM antibodies (Supplemental Figure 8, B and C). In addition, immunofluorescence studies revealed that the CK19+ LPCs were located inside the $\alpha$-SMA-positive fibrotic areas, which is consistent with previous findings (20). In contrast, neither liver fibrosis nor LPC expansion was observed in Ad-Alb-Cre ${ }^{+}$ihCD59 ${ }^{+}$ or Sox9-Cre ${ }^{E R T+} i h C D 59^{+}$mice that received multiple injections of ILY (Supplemental Figure 8A).

Collectively, our results suggest that multiple injections of ILY cause chronic hepatocyte and/or BEC injury in our models, and both hepatocyte and BEC damage can synergistically promote liver fibrosis and LPC expansion in the liver. Finally and importantly, multiple injections of ILY did not induce damage in other tissues (Supplemental Figure 8D), which further supports the specificity of our model.

Application of ihCD59 for neural cell ablation. To demonstrate the applicability of this model for neural cell ablation, we crossed ihCD59 with mGFAPCre mice that expressed Cre recombinase mainly in astrocytes to generate $m G F A P C r e^{+} i h C D 59^{+}$mice. The stereotaxic injection of ILY but not PBS resulted in a dramatic reduction in the number of reactive astrocytes $\left(\mathrm{GFAP}^{+}\right.$and $\left.\mathrm{hCD}^{2} 9^{+}\right)$in the vicinity of the brain injury in $m$ GFAPCre ${ }^{+} i h C D 59^{+}$ but not $i h \mathrm{CD} 9^{+}$mice (Figure 7A). Interestingly, the size of the injury site in $m G F A P C r e^{+} i h C D 59^{+}$mice with specific reactive astrocyte ablation caused by the local injection of ILY was larger than that without astrocyte ablation in the other 3 control groups (PBS- or ILY-treated ihCD59+ mice, PBS-treated mGFAPCre ${ }^{+}$ihCD59 $9^{+}$mice) (Figure 7A). These results suggest that astrocyte ablation at an early stage of injury may prevent the formation of an astrocytic scar, which restricts the expansion of secondary brain injury (4), further indicating that this model is applicable for dissecting the functionality of neural cells. Moreover, i.v. injection of ILY into the mice also mediated the specific ablation of reactive astrocytes, although it was much less efficient than a local injection (Supplemental Figure 9).

In addition, the number of reactive astrocytes was greatly reduced in the $m G F A P C r e^{+} i h C D 59^{+}$mice compared with $i h C D 59^{+}$ mice with ILY treatment upon spinal cord injury (Figure 7B). The recovery of these mice during the postsurgical period was also monitored by examining locomotor function and body weight loss. ILY-treated $m G F A P C r e^{+} i h C D 59^{+}$mice were less active, indicating reduced recovery compared with ILY-treated ihCD59+ mice. Moreover, $m G F A P C r e^{+} i h C D 59^{+}$mice lost $15 \%$ to $22 \%$ of their body weight, whereas ihCD59+ mice only lost approximately $8 \%$ of their body weight after injury, suggesting that ILY-treated $m$ GFAPCre ${ }^{+}$ihCD59 $9^{+}$mice exhibited slower behavioral recovery than did ILY-treated ihCD59+ mice (Supplemental Figure 10). This result may be attributed to the specific local ablation of reactive astrocytes in $m G F A P C r e^{+} i h C D 59^{+}$mice.

A large pharmaceutical window of ILY-mediated cell-ablation model. The acute and chronic toxicity profiles of ILY were examined in Cre-negative ihCD59+ mice by administering $1,500 \mathrm{ng} / \mathrm{g}$ heat-inactivated ILY (no cell lysis activity, Supplemental Figure 11A) or $1500 \mathrm{ng} / \mathrm{g}$ active ILY (10-20 times higher than effective doses $[75-150 \mathrm{ng} / \mathrm{g}]$ used in the ILY-mediated cell-ablation model).
ILY was given for 1 dose to assess acute ILY toxicity or daily for 15 days to assess chronic effects of ILY. As illustrated in Supplemental Figure 11 and Supplemental Figure 12, in ihCD59+ mice treated with 1 dose or 15 doses (for 15 days) of heat-inactivated or active ILY, various blood cell parameters (e.g., the number of white blood cells and various subtypes of blood cells) and serum parameters (e.g., blood urea niutrogen [BUN], creatinine [CREA], amyloid P [AP], ALT, AST, amylase, creatine kinase [CK], and lactate dehydrogenase [LD]) were not altered. In addition, there were no any abnormalities in major organs examined by histological studies in mice with acute or chronic ILY injection. These data indicate that ILY has a pharmacological window that is at least 10- to 20-fold higher than the effective dose required for targeted cell ablation.

\section{Discussion}

In the current study, we successfully developed ihCD59 transgenic mice, which can be used to ablate a wide variety of cell types in vivo by crossing with various lines of Cre transgenic mice and injecting ILY. Compared with several existing cell-ablation models, the ILY/ihCD59 cell-ablation method has several attractive features, including high specificity, a large pharmaceutical window, wide applicability not limited to rodent species, and a rapid ablation mechanism independent of DNA replication or protein synthesis. In addition, we have examined ILY/ihCD59mediated cell ablation in several disease models and demonstrated that this cell-ablation method is valuable for the study of cellular functionalities, tissue injury and regeneration, neural cell functions, etc.

Specificity of cell ablation in a dose-dependent manner without any detectable off-target effects. One limitation of the DT/DTR model is the narrow range of effective doses of DT and its associated significant off-target effects $(5,7,21)$. For example, in the DT/DTR-mediated hepatocyte ablation model (2), the injection of mice with a high dose killed the mice but only induced a mild elevation of serum ALT ( 600 IU/l). The death of the mice was likely caused by off-target effects and not by such mild hepatocyte damage. In contrast, in hepatocytespecific ihCD59 transgenic mice (both $\mathrm{Alb}-\mathrm{Cre} \mathrm{ihCD}^{+} 9^{+}$and Ad-Alb$C r e^{+} i h C D 59^{+}$mice), the injection of ILY caused massive hepatocyte necrosis and substantial elevation of serum ALT ( 8,000 IU/l) without causing mortality, suggesting that ILY specifically lyses hCD59+ hepatocytes in vivo without obvious off-target effects. In addition, we generated several models of hepatic epithelial cell-specific ihCD59 transgenic mice, in which the administration of ILY selectively damaged hepatocytes and/or BECs. The specificity of the ILY/ihCD59 model was also supported by data from the $\mathrm{T}$ cell-ablation model showing that the injection of ILY rapidly ablated CD59+ $\mathrm{T}$ cells in the spleen and peripheral blood of $\mathrm{Lck}-\mathrm{Cr} \mathrm{C}^{+} \mathrm{CD} 59^{+}$mice without affecting B cells, neutrophils, or monocytes. Moreover, toxicity experiments revealed that acute and chronic in vivo treatment with a 10- to 20-fold ILY dose that was higher than the effective dose for cell ablation did not cause any detectable toxicity in Cre-negative ihCD59+ mice, further confirming that ILY has a large pharmaceutical window without any off-target effects. Importantly, we demonstrated that ILY-mediated cell ablation is dose dependent in the $\mathrm{Lck}-\mathrm{Cr}{ }^{+}$ihCD59+ and $\mathrm{Alb}-\mathrm{Cr} \mathrm{C}^{+} \mathrm{ihCD} 59^{+}$mice that we tested. Considering that the high pharmacological window is a key determinant of the therapeutics for clinical usage and of the model system for scientific application, a 
large pharmacological window in our model system will allow us to readily perform the dose-dependent studies to partially or fully ablate the target cells and easily interpret the results as reported here.

Rapid cell ablation: application for the study of cell injury and regeneration. The DT/DTR model $(1,2)$ and the inducible dimerizable caspase-3 model (22) usually require days to achieve efficient cell ablation, resulting in cell injury and regeneration occurring at the same time. It is difficult to use these models to dynamically investigate the responses to acute loss. In contrast, ILY can kill hCD59 ${ }^{+}$cells in vivo within a few minutes for circulating immune cells and within a few hours for cells from intact organs, which allowed us to dynamically investigate their repopulation and regeneration. For example, ILY injection rapidly induced hepatocyte and/or BEC damage in several lines of liver-specific ihCD $59^{+}$mice, followed by induction of the proliferation of these cells, with the peak effects occurring 40 hours after ILY injection. This model is probably the first in which we can selectively induce hepatocyte and/or BEC injury and regeneration. In another model of $\mathrm{Lck}-\mathrm{Cr} \mathrm{e}^{+} \mathrm{ihCD} 59^{+}$mice, the administration of ILY rapidly induced peripheral and spleen $\mathrm{T}$ cell loss without killing thymus T cells; instead, it triggered increased cell proliferation in the medulla of the thymus, a location that is critical for $\mathrm{T}$ cell proliferation and maturation (23). This model will be useful for the study of T cell expansion after acute loss. Finally, we also tested ILY-mediated monocyte and Kupffer cell ablation in Lysm$\mathrm{Cre}^{+} \mathrm{ihCD} 59^{+}$mice and demonstrated that this could be a valuable model for studying the proliferation and repopulation of monocytes and Kupffer cells after their acute loss. The injection of ILY rapidly ablated monocytes in $\mathrm{Lysm}-\mathrm{Cr} \mathrm{e}^{+} \mathrm{ihCD} 59^{+}$mice; however, the kinetics of recovery of blood $\mathrm{Ly}_{6} \mathrm{C}^{+}$and $\mathrm{Ly} 6 \mathrm{C}^{-}$monocytes were different, with much faster $\mathrm{Ly}^{6} \mathrm{C}^{+}$monocyte recovery compared with that of Ly $6 \mathrm{C}^{-}$monocytes, suggesting that Ly $6 \mathrm{C}^{+}$monocytes may serve as precursors of Ly6C- monocytes (24). Additionally, the administration of ILY also rapidly induced the loss of Kupffer cells (liverresident macrophages) and subsequently their proliferation, as indicated by significant BrdU incorporation. Moreover, after adoptive transfer into the ILY-treated Lysm-Cre ${ }^{+} i h C D 59^{+}$mice, in which monocytes and Kupffer cells were depleted, some GFP ${ }^{+}$monocytes acquired the Kupffer cell phenotype (CD11b $\left.{ }^{\text {lo } F} 4 / 80^{\text {hi }}\right)$ (13). Collectively, our findings suggest that circulating monocytes also contribute to Kupffer cell replenishment, in addition to Kupffer cell proliferation, after their acute loss and that the ILY/Lysm-Cre ${ }^{+} i h C D 59^{+}$ model is useful for the study of Kupffer cell replenishment. Finally, we also successfully used ILY/ihCD59-mediated acute cell ablation to study cellular functionalities in 2 models of acute hepatitis, demonstrating a critical role of DCs in $\alpha$-Gal-induced NKT-mediated acute hepatitis and the essential roles of T cells and myeloid cells in Con A-induced acute T cell hepatitis.

Chronic cell ablation in ihCD59 mice after multiple injections of ILY. The unique features of the ILY/ihCD59 model with high specificity and lack of off-target effects make it possible to create a chronic cell-ablation model with multiple injections of ILY. For example, the injection of 3 doses of ILY (daily for 3 days) caused

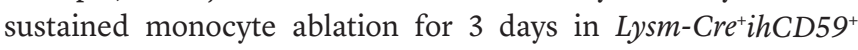
mice. This chronic cell-ablation feature allowed us to study the functions of various types of immune cells in chronic immunemediated diseases, such as EAE, and our findings suggest that $\mathrm{T}$ cells and monocytes play critical pathogenic roles in EAE, a widely used model for human multiple sclerosis (25). At present, there are no effective treatments for multiple sclerosis due to incomplete understanding of its pathogenesis. The roles of various types of immune cells in the pathogenesis of EAE have been previously investigated with different methodologies (25-27), and the therapeutic values targeting each immune cell population have recently gained great attention. In the current study, we compared the functions of several types of immune cells in the pathogenesis of EAE in the same model and demonstrated that T cells play an essential role; monocytes also play a role, but to a lesser extent, in promoting EAE. B cells do not contribute to EAE development.

In addition, we performed multiple ILY injections in 3 lines of hepatic-specific ihCD59 mice, causing chronic damage to hepatocytes and/or BECs. To our surprise, substantial fibrosis and LPC expansion were only observed in multiple ILY-treated $\mathrm{Alb}-\mathrm{Cre} \mathrm{ihCD}^{+} 9^{+}$mice, in which both hepatocytes and BECs were damaged, whereas multiple ILY injections did not cause obvious fibrosis and LPC expansion in Ad-Alb-Cre ${ }^{+} i h C D 59^{+}$mice with only hepatocyte injury and Sox9-Cre ${ }^{E R T+} i h C D 59^{+}$mice with only BEC injury. Collectively, ILY-mediated chronic cell ablation in liverspecific ihCD59 mice is a valuable model for studying liver fibrosis and LPC expansion as caused by hepatocyte and/or BEC injury. Our findings suggest that both hepatocyte and BEC damage are required to efficiently promote liver fibrosis and LPC expansion.

Neural cell ablation. Although we previously reported that ILY was able to pass the blood-brain barrier (11), the i.v. injection of ILY did not efficiently destroy ihCD59+ astrocytes from the brain of $m G F A P^{+} i h C D 59^{+}$mice. This challenge can be overcome by the local injection of ILY or the expression of secretory ILY (sILY) via the viral delivery system. Indeed, in the present study, we demonstrated that the local injection of ILY rapidly induced the death of $m G F A P^{+}$astrocytes in the brain and spinal cord in response to central nervous system injury. This result suggests that neural cells can be efficiently ablated in the ILY/ihCD59 model, but that local ILY injection is required. This rapid neural cell ablation will likely be useful for investigating primary and secondary electrophysiological changes of the neuronal circuits at network levels in vivo and ex vivo upon the ablation of targeted neural and neuronal cells coupled with advanced electrophysiological techniques and optogenetic approaches $(28,29)$. Therefore, this model will likely help us better dissect the neuronal functions/networks and interaction of glial cells and neuronal cells under both physiological and pathogenic conditions.

Other features of ILY/ihCD59. To better utilize the ILY/ihCD59 model for a variety of scientific purposes, several intrinsic features of this model are discussed as follows. First, the presence of cholesterol in the target membrane is required for ILY-mediated pore formation, and the lipid environment of cholesterol in the membrane can affect ILY binding $(8,9,30)$. Interestingly, the addition of exogenous cholesterol $(100-1,600 \mu \mathrm{g} / \mathrm{ml})$ did not influence ILY-mediated cell lysis in vitro (Supplemental Figure 13), suggesting that exogenous cholesterol has minimal effects on ILYmediated cell lysis in hCD59 ${ }^{+}$cells. Although in the present paper, we have shown that injection of ILY efficiently ablated a variety of cell types in several models, it still remains to be determined whether abnormal cholesterol levels and compositions in the blood or in the cellular membrane may affect ILY-mediated lysis 
in vivo, particularly in animal models subjected to specific diets that alter cholesterol levels and/or composition. Second, although we have not fully studied the biodistribution of ILY in mice, we observed that ILY most efficiently ablated circulating leukocytes in peripheral blood and partially deleted leukocytes in the spleen but barely affected the cells in the thymus (data in this study) and bone marrow (data not shown), indicating the existence of ILYprivileged compartments. The potential limitation for using ILY in these compartments could be overcome via the local injection. Nevertheless, the biodistribution of ILY requires further investigation. Third, it has been well documented that ILY binds to hCD59 on cell membrane and subsequently induces pore formation and cell necrosis (9). ILY induction of cell necrosis in several lines of $\mathrm{Cre}^{+} i h \mathrm{CD} 59^{+}$mice is also demonstrated in the present study, such as hepatocyte necrosis in H\&E staining, as shown in Supplemental Figure 7, and uptake of a fluorescent reactive dye in the flow cytometry analysis shown in Figure 1E. Fourth, cell death (necrosis or apoptosis) is a well-documented factor in inducing sterile inflammation, which may occur to different extents in various cell-ablation models, including the ILY/ihCD59 model described in the current study. In general, sterile inflammation is weak and transient in the majority of the ILY/hCD59-mediated cell-ablation models, in which a small number of cells are depleted, which does not result in death of hCD59-negative cells. For example, injection of ILY killed approximately $96 \%$ of hCD59 ${ }^{+} \mathrm{T}$ cells but did not affect hCD59-negative B cells in $L c k-C r e^{+} h C D 59^{+}$mice, as demonstrated in the current study. Therefore, ILY-mediated cell death provoked a mild to moderate but transient inflammatory response in the majority of the ILY/ihCD59 models, making this model have wide application potential for studying cellular functionality in vivo and investigating the pathogenesis of human diseases. However, if ILY injection induces massive cell death in some ihCD59 models, such as Alb-Cre ${ }^{+} i h C D 59^{+}$mice, strong sterile inflammation may occur. An example from the current study is the ILY injection in $\mathrm{Alb}-\mathrm{Cr} e^{+} h \mathrm{CD} 59^{+}$mice, which causes massive hepatocyte necrosis and subsequent induction of strong sterile inflammation (31). Strong sterile inflammatory response in the $\mathrm{ILY} / \mathrm{Alb}-\mathrm{Cre}{ }^{+} h \mathrm{CD} 59^{+}$mice is not a limitation, but is rather a more specific model for studying how specific hepatocyte death induces sterile inflammation and how this sterile inflammation modulates liver injury and regeneration compared with many other models of liver sterile inflammation induced by drugs, ischemia/reperfusion, and alcoholic and nonalcoholic steatohepatitis that not only involve hepatocyte injury but also include metabolism, hypoxia, endotoxin, etc. (31).

In summary, we have developed and characterized an important and versatile tool that can be used to study the effects of cell ablation in any organ system or cell type and therefore has universal application and value to investigators in multiple fields. This approach can be used to study the functions of individual cell populations in multiple disease models and to investigate tissue injury and regeneration.

\section{Methods}

Generation of ihCD59 mice and ILY purification. Generation of ihCD59 mice and ILY purification are detailed in Supplemental Experimental Procedures.
Flow cytometry analysis. Single-cell suspension was adjusted to 1 $\times 10^{6}$ cell per $100 \mu \mathrm{l}$ staining buffer and incubated with hCD59 (clone OV9A2), CD19 (clone eBio1D3), CD8a (clone 53-6.7), CD4 (clone GK1.5), CD11c (clone N418), and CD3 (clone 145-2C11) antibodies (eBioscience) for 30 minutes at $4^{\circ} \mathrm{C}$ in the dark. For dead cell staining, cells with surface staining were stained with DAPI. Flow cytometry data were obtained from BD FACSCalibur and analyzed by FlowJo software (Tree Star Inc.).

Adoptive transfer of monocytes. Bone marrow cells and peripheral blood mononuclear cell (PBMCs) were collected from $\mathrm{GFP}^{+}$mice. Monocytes were purified by negative selection using the MACS Monocyte Isolation Kit (Miltenyi Biotec). Purified monocytes $\left(7.5 \times 10^{6}\right)$ with $100 \mathrm{ng} / \mathrm{g}$ ILY were injected into $\mathrm{Lysm}-\mathrm{Cr} \mathrm{e}^{+} i \mathrm{C} \mathrm{CD} 59^{+}$mice by i.v. injection. Mice were sacrificed 3 days later, and Kupffer cells were isolated and analyzed.

Kupffer cell isolation and analysis. Kupffer cells were analyzed by flow cytometry as described previously, with minor modifications (13). Briefly, livers were perfused with EGTA buffer and digested with GBSS containing collagenase. Nonparenchymal cells were obtained by removing hepatocytes using 35\% Percoll density centrifugation. Nonparenchymal cells were further stained with CD45 (clone 30-F11), F4/80 (clone BM8), CD11b (clone M1/70), and Gr-1 (clone RB6-8C5) antibodies for flow cytometry analysis.

Con A-induced liver injury, EAE, CNS injury models, ILY injections, biochemical analysis, and immunostaining analysis. Detailed methods are described in Supplemental Experimental Procedures.

Statistics. Data are expressed as mean \pm SD. To compare values obtained from 3 or more groups, 1-way ANOVA was used, followed by Dunnett's post-hoc test. To compare multiple groups over time, 2-way ANOVA followed by Bonferroni's post-hoc test was used. To compare values obtained from 2 groups, 2-tailed Student's $t$ test was performed. Statistical significance was defined as $P<0.05$.

Study approval. The NIAAA Animal Care and Use Committee approved all animal studies.

\section{Author contributions}

$\mathrm{DF}, \mathrm{SD}$, and FL performed experiments, analyzed data, and wrote the paper. YO, ZZ, Hua Wang, YGZ, AK, XP, FZ, CZ, MC, UH, ML, $\mathrm{YH}$, and MX performed experiments. LZ, Hong Wang, XY, CJ, ECB, JG, KK, WH, and SL analyzed data. XQ and BG supervised the project, designed experiments, analyzed data, and wrote the paper.

\section{Acknowledgments}

This work was supported by the intramural program of NIAAA (to B. Gao), NIH 1R01CA166144 and RO1 AI061174 (to X. Qin), China Scholarship 201306220151 (to S. Dai), Major Research Plan of the National Natural Science Foundation of China 91439124 (to L. Zhang), Shangdong Province Taishan Scholar Project of China, core facilities grant for the Comprehensive NeuroAIDS Center (CNAC NIMH P30MH092177) (to K. Khalili), and NIH1R01 NS079432 and 1R01 EY024575, and Shriners Research Foundation (SHC-86300) grants (to S. Li). A. Kearns was supported by NIH under a Ruth L. Kirschstein National Research Service Award (5T32MH079785). We thank David Lovinger and Li Zhang (NIAAA, NIH) for advice on the neural cell-ablation study and Chuxia Deng (NIDDK, NIH) for advice on the generation of the ihCD59 construct. We also thank Liangyou Rui (University of Michigan, Ann Arbor, Michigan, USA) for providing the Alb-Cre adenovirus. 
Address correspondence to: Bin Gao, Laboratory of Liver Diseases, NIAAA/NIH, 5625 Fishers Lane, Bethesda, Maryland 20892, USA. Phone:301.443.3998; E-mail: bgao@mail.nih.gov. Or to:Xuebin Qin,
Department of Neuroscience, Temple University School of Medicine, Room 749, 3500 N Broad Street, Philadelphia, Pennsylvania 19140, USA. Phone: 215.707.5823; E-mail: xuebin.qin@temple.edu.
1. Buch T, et al. A Cre-inducible diphtheria toxin receptor mediates cell lineage ablation after toxin administration. Nat Methods. 2005;2(6):419-426.

2. Saito M, et al. Diphtheria toxin receptor-mediated conditional and targeted cell ablation in transgenic mice. Nat Biotechnol. 2001;19(8):746-750.

3. Minasi LE, Kamogawa Y, Carding S, Bottomly K, Flavell RA. The selective ablation of interleukin 2-producing cells isolated from transgenic mice. JExp Med. 1993;177(5):1451-1459.

4. Burda JE, Sofroniew MV. Reactive gliosis and the multicellular response to CNS damage and disease. Neuron. 2014;81(2):229-248.

5. Chapman TJ, Georas SN. Adjuvant effect of diphtheria toxin after mucosal administration in both wild type and diphtheria toxin receptor engineered mouse strains. JImmunol Methods. 2013;400-401:122-126.

6. Christiaansen AF, Boggiatto PM, Varga SM. Limitations of Foxp3(+) Treg depletion following viral infection in DEREG mice. J Immunol Methods. 2014;406:58-65.

7. Goldwich A, Steinkasserer A, Gessner A, Amann K. Impairment of podocyte function by diphtheria toxin - a new reversible proteinuria model in mice. Lab Invest. 2012;92(12):1674-1685.

8. Nagamune H, et al. Intermedilysin, a novel cytotoxin specific for human cells secreted by Streptococcus intermedius UNS46 isolated from a human liver abscess. Infect Immun. 1996;64(8):3093-3100.

9. Giddings KS, Zhao J, Sims PJ, Tweten RK. Human CD59 is a receptor for the cholesterol-dependent cytolysin intermedilysin. Nat Struct Mol Biol. 2004;11(12):1173-1178.

10. Kimberley FC, Sivasankar B, Paul Morgan B. Alternative roles for CD59. Mol Immunol. 2007;44(1-3):73-81.

11. $\mathrm{Hu} \mathrm{W}$, et al. Rapid conditional targeted ablation of cells expressing human CD59 in transgenic mice by intermedilysin. Nat Med. 2008;14(1):98-103.
12. $\mathrm{Hu} \mathrm{W}$, et al. The critical roles of platelet activation and reduced NO bioavailability in fatal pulmonary arterial hypertension in a murine hemolysis model. Blood. 2010;116(9):1613-1622.

13. Holt MP, Cheng L, Ju C. Identification and characterization of infiltrating macrophages in acetaminophen-induced liver injury. J Leukoc Biol. 2008;84(6):1410-1421.

14. Wang H, Feng D, Park O, Yin S, Gao B. Invariant NKT cell activation induces neutrophil accumulation and hepatitis: opposite regulation by IL-4 and IFN- $\gamma$. Hepatology. 2013;58(4):1474-1485.

15. Tiegs G. Cellular and cytokine-mediated mechanisms of inflammation and its modulation in immune-mediated liver injury. $Z$ Gastroenterol. 2007;45(1):63-70.

16. Constantinescu CS, Farooqi N, O'Brien K, Gran B. Experimental autoimmune encephalomyelitis (EAE) as a model for multiple sclerosis (MS). Br J Pharmacol. 2011;164(4):1079-1106.

17. $\mathrm{Li} \mathrm{H}$, et al. PI3K $\gamma$ inhibition alleviates symptoms and increases axon number in experimental autoimmune encephalomyelitis mice. Neuroscience. 2013;253:89-99.

18. Postic C, et al. Dual roles for glucokinase in glucose homeostasis as determined by liver and pancreatic $\beta$ cell-specific gene knock-outs using Cre recombinase. J Biol Chem. 1999;274(1):305-315.

19. Tarlow BD, Finegold MJ, Grompe M. Clonal tracing of Sox $9^{+}$liver progenitors in mouse oval cell injury. Hepatology. 2014;60(1):278-289.

20. Boulter L, et al. Macrophage-derived Wnt opposes Notch signaling to specify hepatic progenitor cell fate in chronic liver disease. Nat Med 2012;18(4):572-579.

21. Christiaansen AF, Boggiatto PM, Varga SM. Limitations of Foxp3 Treg depletion following viral infection in DEREG mice. JImmunol Methods. 2014;406:58-65.

22. Mallet VO, et al. Conditional cell ablation by tight control of caspase-3 dimerization in transgenic mice. Nat Biotechnol. 2002;20(12):1234-1239.

23. Takahama Y. Journey through the thymus: stromal guides for T-cell development and selection. Nat Rev Immunol. 2006;6(2):127-135.

24. Lin SL, Castano AP, Nowlin BT, Lupher ML Jr, Duffield JS. Bone marrow Ly6Chigh monocytes are selectively recruited to injured kidney and differentiate into functionally distinct populations. JImmunol. 2009;183(10):6733-6743.

25. Fletcher JM, Lalor SJ, Sweeney CM, Tubridy N, Mills KH. T cells in multiple sclerosis and experimental autoimmune encephalomyelitis. Clin Exp Immunol. 2010;162(1):1-11.

26. Ajami B, Bennett JL, Krieger C, McNagny $\mathrm{KM}$, Rossi FM. Infiltrating monocytes trigger EAE progression, but do not contribute to the resident microglia pool. Nat Neurosci. 2011;14(9):1142-1149.

27. Matsushita T, Yanaba K, Bouaziz JD, Fujimoto M, Tedder TF. Regulatory B cells inhibit EAE initiation in mice while other B cells promote disease progression. J Clin Invest. 2008;118(10):3420-3430.

28. Kuang SY, et al. Prolonging life in chick forebrainneuron culture and acquiring spontaneous spiking activity on a microelectrode array. Biotechnol Lett. 2015;37(3):499-509.

29. Witten IB, et al. Recombinase-driver rat lines: tools, techniques, and optogenetic application to dopamine-mediated reinforcement. Neuron. 2011;72(5):721-733.

30. Tweten RK, Hotze EM, Wade KR. The unique molecular choreography of giant pore formation by the cholesterol-dependent cytolysins of gram-positive bacteria. Annu Rev Microbiol. 2015;69:323-340.

31. Kubes P, Mehal WZ. Sterile inflammation in the liver. Gastroenterology. 2012;143(5):1158-1172.

32. Keith BJF, George P. The mouse brain in stereotaxic coordinates. Compact 3rd ed. Amsterdam: Elsevier Academic Press. 2008:plate 29. 